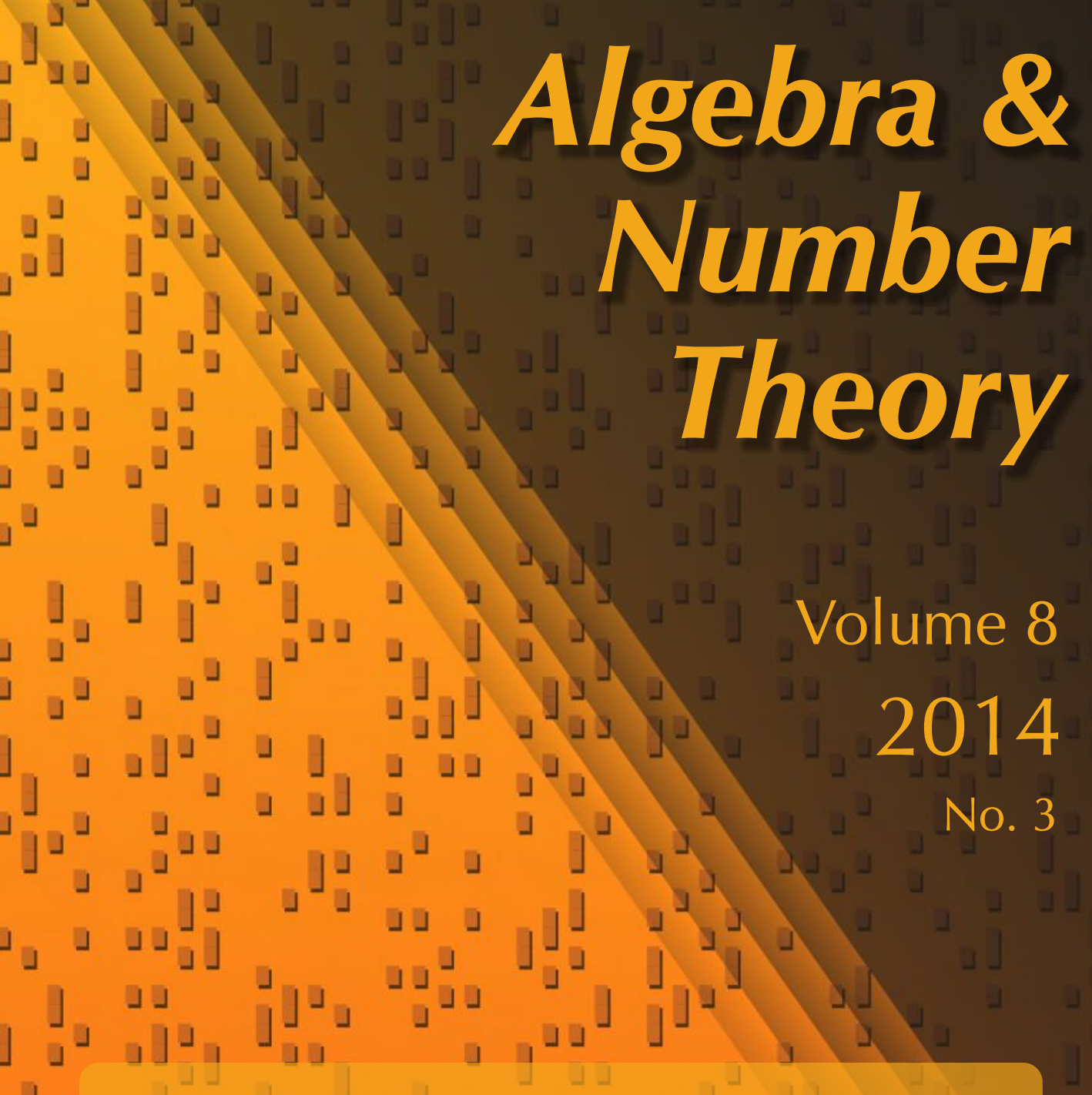




\title{
Linear forms in logarithms and integral points on higher-dimensional varieties
}

\author{
Aaron Levin
}

\begin{abstract}
We apply inequalities from the theory of linear forms in logarithms to deduce effective results on $S$-integral points on certain higher-dimensional varieties when the cardinality of $S$ is sufficiently small. These results may be viewed as a higher-dimensional version of an effective result of Bilu on integral points on curves. In particular, we prove a completely explicit result for integral points on certain affine subsets of the projective plane. As an application, we generalize an effective result of Vojta on the three-variable unit equation by giving an effective solution of the polynomial unit equation $f(u, v)=w$, where $u, v$, and $w$ are $S$-units, $|S| \leq 3$, and $f$ is a polynomial satisfying certain conditions (which are generically satisfied). Finally, we compare our results to a higher-dimensional version of Runge's method, which has some characteristics in common with the results here.
\end{abstract}

\section{Introduction}

The problem of proving effective results in Diophantine questions is one of the most pervasive and basic problems in number theory. Already in the case of curves, the fundamental finiteness theorems for integral points and rational points (Siegel's theorem and Faltings' theorem, respectively) are not known in an effective way, that is, in general there is no known algorithm to provably compute the finite sets in the conclusion of either theorem. In certain special cases, however, effective techniques have been developed for computing integral or rational points on curves. The most general and widely used effective methods for integral points on curves come from the theory of linear forms in logarithms, developed originally by Baker [1975]. In higher dimensions, effective techniques have not received much attention. A natural first step towards proving higher-dimensional effective results consists of taking the known effective techniques for curves and applying them, to the extent possible, to the higher-dimensional situation. In [Levin 2008], some progress towards this goal was achieved by formulating a higher-dimensional version of an effective

Research partially supported by NSF grant DMS-1102563.

MSC2010: primary 11G35; secondary 11J86, $11 \mathrm{D} 61$.

Keywords: integral points, unit equation, linear forms in logarithms, Runge's method. 
method of Runge for computing integral points on curves. In this article we will consider the theory of linear forms in logarithms and applications to integral points on higher-dimensional varieties.

One of the few directions in which progress has been made on the study of integral points on higher-dimensional varieties involves varieties which, roughly speaking, have many components at infinity (see, for example, [Autissier 2009; 2011; Corvaja et al. 2009; Corvaja and Zannier 2004; 2006; Levin 2008; 2009]). The results given here also fit into this framework. We prove the following effective result for integral points on higher-dimensional varieties.

Theorem 1. Let $X$ be a nonsingular projective variety defined over a number field $k$. Let $D_{1}, \ldots, D_{n}$ be effective ample divisors on $X$ defined over $k$. Let $D=\sum_{i=1}^{n} D_{i}$. Let $m \leq n$ be a positive integer such that for all subsets $I \subset\{1, \ldots, n\},|I|=m$, the set $\bigcap_{i \in I}\left(\operatorname{Supp} D_{i}\right)(\bar{k})$ consists of finitely many points. Suppose that for each point $P \in(\operatorname{Supp} D)(\bar{k})$, there exists a nonconstant rational function $\phi \in k(X)$ satisfying $P \notin \operatorname{Supp} \phi$ and $\operatorname{Supp} \phi \subset \operatorname{Supp} D$. Let $S$ be a set of places of $k$ containing the archimedean places with

$$
(m-1)|S|<n .
$$

Let $R$ be a set of $S$-integral points on $X \backslash D$. Suppose that $X, D_{1}, \ldots, D_{n}, D, R, S, k$ satisfy (*) in Section 3.2. Then $R$ is contained in an effectively computable proper closed subset $Z$ of $X$.

To make the meaning of "effective" precise, we have assumed in the theorem that one can compute certain natural quantities described in Section 3.2. An explicit description of the higher-dimensional part of $Z$ is given in Theorem 14. If $X=C$ is a curve, then Theorem 1 (with $m=1$ ) is easily seen to be equivalent to the following theorem of Bilu.

Theorem 2 [Bilu 1995]. Let $C \subset \mathbb{A}^{n}$ be an affine curve defined over a number field $k$. Suppose that there exist two everywhere nonvanishing regular functions on $C$ with multiplicatively independent images in $k(C)^{*} / k^{*}$. For any finite set of places $S$ of $k$ containing the archimedean places, the set $C\left(\mathrm{O}_{k, S}\right)$ is finite and effectively computable.

Thus, Theorem 1 may be viewed as a higher-dimensional generalization of Bilu's theorem. We note that, as mentioned in [Bilu 1995], when combined with finite covers and the Chevalley-Weil theorem, Theorem 2 appears to be responsible for all known "universally effective" results on integral points on curves (results valid for all number fields $k$ and finite sets of places $S$ ).

As an easy consequence of Theorem 1, we obtain the following result for integral points on surfaces. 
Corollary 3. Let $X$ be a nonsingular projective surface defined over a number field $k$. Let $D_{1}, \ldots, D_{n}$ be ample effective divisors on $X$, defined over $k$, that generate a subgroup of $\operatorname{Pic}(X)$ of rank $r$ and pairwise do not have any common components. Let $D=\sum_{i=1}^{n} D_{i}$. Suppose that the intersection of the supports of any $n-r$ of the divisors $D_{i}$ is empty. Let $S$ be a set of places of $k$ containing the archimedean places with

$$
|S|<n \text {. }
$$

Let $R$ be a set of $S$-integral points on $X \backslash D$. Suppose that $X, D_{1}, \ldots, D_{n}, D, R, S, k$ satisfy (*) in Section 3.2. Then $R$ is contained in an effectively computable proper closed subset $Z$ of $X$.

The requirement, in the above results, that the number of components at infinity be large relative to the cardinality of $S$ appears prominently in Runge's method [Levin 2008] as well. We will compare our results with a higher-dimensional version of Runge's method in Section 5.

As an application of our result on surfaces, we prove an effective result on two-variable polynomials that take on $S$-unit values at $S$-unit arguments when $|S| \leq 3$.

Corollary 4. Let $f \in k[x, y]$ be a polynomial of degree $d>0$ such that $f(0,0) \neq 0$ and $x^{d}$ and $y^{d}$ have nonzero coefficients in $f$. Let $S$ be a finite set of places of $k$ containing the archimedean places with $|S| \leq 3$. Then the set of solutions to

$$
f(u, v)=w, \quad u, v, w \in \mathbb{O}_{k, S}^{*},
$$

consists of a finite effectively computable set and a finite number of infinite families of solutions where one of $u, v$, or $w$ is constant.

The infinite families of solutions are explicitly described in Corollary 21 in Section 4.

Taking $f(x, y)$ to be an appropriate affine linear polynomial, we find that Corollary 4 generalizes an effective result of Vojta on the three-variable $S$-unit equation with $|S| \leq 3$.

Theorem 5 [Vojta 1983]. Let $k$ be a number field, $S$ a finite set of places of $k$ containing the archimedean places, and $a_{1}, a_{2}, a_{3} \in k^{*}$. If $|S| \leq 3$, then the set of solutions to the equation

$$
a_{1} u_{1}+a_{2} u_{2}+a_{3} u_{3}=1, \quad u_{1}, u_{2}, u_{3} \in \mathbb{O}_{k, S}^{*},
$$

with

$$
\sum_{i \neq j} a_{i} u_{i} \neq 0, \quad j=1,2,3
$$

is finite and effectively computable. 
We note that versions of Theorem 5 with $k=\mathbb{Q}$ were also proved by Mo and Tijdeman [1992] and Skinner [1990]. Ineffectively, versions of Corollary 4 and Theorem 5 can be proven without any assumption on the (finite) cardinality of $S$. For Theorem 5, this is a special case of a well-known result on unit equations, proved independently by Evertse [1984] and van der Poorten and Schlickewei [1982]. In the case of Corollary 4, this is an easy consequence of a result of Vojta [1987, Corollary 2.4.3] and the proof of Corollary 4. The ineffectivity here comes ultimately from usage of the Schmidt subspace theorem.

More generally, Vojta proved the following result for systems of unit equations.

Theorem 6 (Vojta). Let $m$ and $n$ be positive integers with $n>m$. Let $\left(a_{i j}\right)$ be an $m \times n$ matrix with elements in a number field $k$ such that no $m+1$ distinct columns of the matrix have rank less than $m$, and such that no column is identically zero. Assume further that $S$ is a finite set of places of $k$, containing the archimedean places, satisfying

$$
(n-m-2)|S|<n .
$$

Then the set of solutions to the system of unit equations

$$
a_{i 1} u_{1}+\cdots+a_{i n} u_{n}=0, \quad 1 \leq i \leq m, \quad u_{1}, \ldots, u_{n} \in \mathbb{O}_{k, S}^{*},
$$

can be effectively determined.

More precisely, viewing a solution in Theorem 6 as a point in $\mathbb{P}^{n-1}$, the set of solutions to a system of equations as in Theorem 6 lies in finitely many proper linear subspaces of $\mathbb{P}^{n-1}$, and these solutions may be explicitly described and parametrized. In work to appear, Bennett (personal communication) has improved the inequality on $|S|$ in Theorem 6 to $(n-m-1)|S|<2 n$. In particular, Bennett's methods allow one to extend Theorem 5 to four-variable unit equations, that is, to effectively solve the unit equation

$$
a_{1} u_{1}+a_{2} u_{2}+a_{3} u_{3}+a_{4} u_{4}=1, \quad u_{1}, u_{2}, u_{3}, u_{4} \in \mathrm{O}_{k, S}^{*},
$$

where $a_{1}, a_{2}, a_{3}, a_{4} \in k^{*}$ and $|S| \leq 3$. It would be interesting to determine the extent to which Bennett's methods may be applied to gain a similar improvement to the results presented here.

In Section 7, we prove a completely explicit version of Corollary 3 when $X=\mathbb{P}^{2}$ is the projective plane.

Theorem 7. Let $k$ be a number field of degree $\delta$ and discriminant $\Delta$. Let $C_{1}, \ldots, C_{n}$ be distinct curves over $k$ in $\mathbb{P}^{2}$ such that the intersection of any $n-1$ of the curves is empty. Let $S$ be a set of places of $k$ containing the archimedean places with $s=|S|<n$. Then the set of integral points $\left(\mathbb{P}^{2} \backslash \bigcup_{i=1}^{n} C_{i}\right)\left(\mathscr{O}_{k, S}\right)$ is contained in an effectively computable proper Zariski closed subset $Z$ of $\mathbb{P}^{2}$. Explicitly, let 
$d_{i}=\operatorname{deg} C_{i}, d=\max _{i} d_{i}, h=\max _{i} h\left(C_{i}\right)$, and $N=\max _{v \in S} N(v)$. Let $C_{i}$ be defined by $f_{i} \in k[x, y, z], i=1, \ldots, n$. Let $T=\bigcup_{i \neq j}\left(C_{i} \cap C_{j}\right)(\bar{k})$, and for each point $P \in T$, let $I_{P}=\left\{i: P \notin C_{i}\right\}$. For $P \in T$, let

$$
\Phi_{P}=\left\{\frac{f_{i}^{d_{j}}}{f_{j}^{d_{i}}}: i, j \in I_{P}\right\} .
$$

Then $Z$ may be taken to consist of the union of the finite set of points

$$
\begin{aligned}
& \{P \in X(k): \\
& \left.\quad h(P)<2^{20 s+4 \delta+75} d^{6 s+34} \delta^{5 s+8 \delta-3} s^{4 s+2} N^{d^{2}}\left(\log ^{*} N\right)^{2 s}|\Delta|^{3 / 2}\left(\log ^{*}|\Delta|\right)^{3 \delta}(h+1)\right\}
\end{aligned}
$$

and the Zariski closure $Z^{\prime}$ of the set

$$
\bigcup_{P \in T} \bigcap_{\phi \in \Phi_{P}}\{Q \in X(\bar{k}): \phi(Q)=\phi(P)\} .
$$

Being more interested in the general shape of the explicit height bound in the theorem, we have made no effort here to obtain the best possible explicit bound coming from the proof of Theorem 7 (and indeed, carefully following the proof gives a superior, but more cumbersome, expression).

Finally, we give a brief sketch of the proof of Theorem 1. The proof is a generalization of the proofs of Bilu's and Vojta's results (Theorem 2 and Theorem 5). Let $R$ be a set of $S$-integral points on $X \backslash D$, as in Theorem 1, and let $P \in R$. Let $T \subset X(\bar{k})$ be the finite set of points contained in the support of $m$ or more divisors $D_{i}$. Using the assumption on the cardinality of $S$, the pigeonhole principle implies that for some point $Q \in T$ and $v \in S, P$ is $v$-adically close to $Q$. Our hypotheses then provide us with a nonconstant rational function $\phi \in k(X)$ with zeros and poles only in Supp $D \backslash\{Q\}$. Since $P \in R, \phi(P)$ is essentially an $S$-unit, and $\phi(P)$ is $v$-adically close to $\phi(Q)$. Now assuming that $\phi(P) \neq \phi(Q)$ (this is where a higher-dimensional exceptional set may appear), we apply a Baker-type inequality to conclude that $\phi(P)$, and hence $P$, must have height bounded by an explicit constant.

\section{Notation and definitions}

Let $k$ be a number field and let $S$ be a finite set of places of $k$ containing the archimedean places. We use $\mathrm{O}_{k}, \mathrm{O}_{k, S}$, and $\mathrm{O}_{k, S}^{*}$ to denote the ring of integers of $k$, ring of $S$-integers of $k$, and group of $S$-units of $k$, respectively. Throughout, we let $\delta=[k: \mathbb{Q}]$ be the degree of $k, \Delta$ the (absolute) discriminant of $k, R_{k}$ the regulator of $k$, and $R_{S}$ the $S$-regulator.

Recall that we have a canonical set $M_{k}$ of places (or absolute values) of $k$ consisting of one place for each prime ideal $\mathfrak{p}$ of $\mathcal{O}_{k}$, one place for each real 
embedding $\sigma: k \rightarrow \mathbb{R}$, and one place for each pair of conjugate embeddings $\sigma, \bar{\sigma}: k \rightarrow \mathbb{C}$. For $v \in M_{k}$, we define

$$
N(v)= \begin{cases}2 & \text { if } v \text { is archimedean, } \\ N(\mathfrak{p}) & \text { if } v \text { corresponds to the prime } \mathfrak{p},\end{cases}
$$

where $N(\mathfrak{p})=\left|\mathscr{O}_{k} / \mathfrak{p}\right|$ is the norm of $\mathfrak{p}$. We normalize our absolute values so that $|p|_{v}=1 / p$ if $v$ corresponds to $\mathfrak{p}$ and $\mathfrak{p}$ lies above a rational prime $p$, and $|x|_{v}=|\sigma(x)|$ if $v$ corresponds to an embedding $\sigma$. For $v \in M_{k}$, let $k_{v}$ denote the completion of $k$ with respect to $v$. We set

$$
\|x\|_{v}=|x|_{v}^{\left[k_{v}: \mathbb{Q}_{v}\right] /[k: \mathbb{Q}]} .
$$

A fundamental equation is the product formula

$$
\prod_{v \in M_{k}}\|x\|_{v}=1
$$

which holds for all $x \in k^{*}$.

For $x$ a positive real number we let

$$
\begin{aligned}
& \log ^{*} x=\max \{\log x, 1\}, \\
& \epsilon_{v}(x)= \begin{cases}x & \text { if } v \text { is archimedean } \\
1 & \text { otherwise }\end{cases}
\end{aligned}
$$

and

$$
\epsilon_{v}^{\prime}(x)=\epsilon_{v}(x)^{\left[k_{v}: \mathbb{Q} v\right] /[k: \mathbb{Q}]} .
$$

We note that

$$
\prod_{v \in M_{k}} \epsilon_{v}^{\prime}(x)=x .
$$

In this notation, for $v \in M_{k}$ and $x, y \in k$ we have the inequalities

$$
\begin{aligned}
|x+y|_{v} & \leq \epsilon_{v}(2) \max \left\{|x|_{v},|y|_{v}\right\}, \\
\|x+y\|_{v} & \leq \epsilon_{v}^{\prime}(2) \max \left\{\|x\|_{v},\|y\|_{v}\right\} .
\end{aligned}
$$

For $v \in M_{k}$ and $\alpha \in k$, we define the local height

$$
h_{v}(\alpha)=\log \max \left\{\|\alpha\|_{v}, 1\right\}
$$

and the height

$$
h(\alpha)=\sum_{v \in M_{k}} h_{v}(\alpha)
$$


We will frequently make the identification $\mathbb{P}^{1}(k)=k \cup\{\infty\}$. More generally, for a point $P=\left(x_{0}, \ldots, x_{n}\right) \in \mathbb{P}^{n}(k)$, we have the absolute logarithmic height

$$
h(P)=\sum_{v \in M_{k}} \log \max \left\{\left\|x_{0}\right\|_{v}, \ldots,\left\|x_{n}\right\|_{v}\right\}
$$

Note that this is independent of the number field $k$ and the choice of coordinates $x_{0}, \ldots, x_{n} \in k$.

For a polynomial $f \in k\left[x_{1}, \ldots, x_{n}\right]$ and $v \in M_{k}$, we let $|f|_{v}$ denote the maximum of the absolute values of the coefficients of $f$ with respect to $v$. We define $\|f\|_{v}$ similarly. We define the height of a nonzero polynomial by

$$
h(f)=\sum_{v \in M_{k}} \log \|f\|_{v}
$$

This is the same as the height of the point in projective space whose coordinates are given by the coefficients of $f$. If $\phi: \mathbb{P}^{n} \rightarrow \mathbb{P}^{m}$ is a rational map, where $\phi=\left(f_{0}, \ldots, f_{m}\right)$ and $f_{0}, \ldots, f_{m} \in k\left[x_{0}, \ldots, x_{n}\right]$ are polynomials with no common factor, then we define

$$
h(\phi)=\sum_{v \in M_{k}} \log \max _{i}\left\|f_{i}\right\|_{v}
$$

Let $D$ be a hypersurface in $\mathbb{P}^{n}$ defined by a homogeneous polynomial $f \in$ $k\left[x_{0}, \ldots, x_{n}\right]$ of degree $d$. We define

$$
h(D)=h(f) .
$$

For $v \in M_{k}$ and $P=\left(x_{0}, \ldots, x_{n}\right) \in \mathbb{P}^{n}(k) \backslash D, x_{0}, \ldots, x_{n} \in k$, we define the local height function

$$
h_{D, v}(P)=\log \frac{\|f\|_{v} \max _{i}\left\|x_{i}\right\|_{v}^{d}}{\|f(P)\|_{v}} .
$$

Note that this definition is independent of the choice of the defining polynomial $f$ and the choice of the coordinates for $P$. We let $h_{D}(P)=(\operatorname{deg} D) h(P)$. By the product formula, if $P \in \mathbb{P}^{n}(k) \backslash D$, then $\sum_{v \in M_{k}} h_{D, v}(P)=h_{D}(P)$.

If $P=\left(x_{0}, \ldots, x_{n}\right), Q=\left(y_{0}, \ldots, y_{n}\right) \in \mathbb{P}^{n}(k), x_{i}, y_{i} \in k, P \neq Q$, and $v \in M_{k}$, we define

$$
h_{Q, v}(P)=\log \frac{\max _{i}\left\|x_{i}\right\|_{v} \max _{i}\left\|y_{i}\right\|_{v}}{\max _{i, j}\left\|x_{i} y_{j}-x_{j} y_{i}\right\|_{v}} .
$$

Much more generally, one can associate a height to any closed subscheme of a projective variety. We give here a quick summary of the relevant properties of such heights and refer the reader to [Silverman 1987] for the general theory and details.

Let $Y$ be a closed subscheme of a projective variety $X$, both defined over $k$. For $v \in M_{k}$, one can associate a local height function $h_{Y, v}: X(k) \backslash Y \rightarrow \mathbb{R}$, well-defined up to $O(1)$, and a global height function $h_{Y}$, well-defined up to $O(1)$, which is 
a sum of appropriate local height functions. If $Y=D$ is an effective (Cartier) divisor (which we will frequently identify with the associated closed subscheme), these height functions agree with the usual height functions associated to divisors. Local height functions satisfy the following properties: if $Y$ and $Z$ are two closed subschemes of $X$, defined over $k$, and $v \in M_{k}$, then up to $O(1)$,

$$
\begin{aligned}
h_{Y \cap Z, v} & =\min \left\{h_{Y, v}, h_{Z, v}\right\}, & & \\
h_{Y+Z, v} & =h_{Y, v}+h_{Z, v}, & & \\
h_{Y, v} & \leq h_{Z, v} & & \text { if } Y \subset Z, \\
h_{Y, v} & \leq c h_{Z, v} & & \text { if } \operatorname{Supp} Y \subset \operatorname{Supp} Z,
\end{aligned}
$$

for some constant $c>0$, where $\operatorname{Supp} Y$ denotes the support of $Y$. If $\phi: W \rightarrow X$ is a morphism of projective varieties, then

$$
h_{Y, v}(\phi(P))=h_{\phi^{*} Y, v}(P) \quad \text { for all } P \in W(k) \backslash \phi^{*} Y .
$$

Here, $Y \cap Z, Y+Z, Y \subset Z$, and $\phi^{*} Y$ are defined in terms of the associated ideal sheaves (see [Silverman 1987]). Global height functions satisfy similar properties (except the first property above, which becomes $h_{Y \cap Z} \leq \min \left\{h_{Y}, h_{Z}\right\}+O(1)$ ).

Let $D$ be a divisor on a nonsingular projective variety $X$. For a nonzero rational function $\phi \in \bar{k}(X)$, we let $\operatorname{div}(\phi)$ denote the divisor associated to $\phi$. We let Supp $D$ denote the support of $D$ and $\operatorname{Supp} \phi=\operatorname{Supp} \operatorname{div}(\phi)$. Let

$$
L(D)=\{\phi \in \bar{k}(X): \operatorname{div}(\phi)+D \geq 0\}
$$

and $h^{0}(D)=\operatorname{dim} H^{0}(X, \mathcal{O}(D))=\operatorname{dim} L(D)$. If $h^{0}(n D)=0$ for all $n>0$, then we let $\kappa(D)=-\infty$. Otherwise, we define the dimension of $D$ to be the integer $\kappa(D)$ such that there exist positive constants $c_{1}$ and $c_{2}$ with

$$
c_{1} n^{\kappa(D)} \leq h^{0}(n D) \leq c_{2} n^{\kappa(D)}
$$

for all sufficiently divisible $n>0$. We define a divisor $D$ on $X$ to be big if $\kappa(D)=\operatorname{dim} X$.

Let $D$ be an effective divisor on $X$ and $h_{D}=\sum_{v \in M_{k}} h_{D, v}$ a height function associated to $D$. A set of points $R \subset X(k) \backslash D$ is called a set of $S$-integral points on $X \backslash D$ if there exist constants $c_{v}, v \in M_{k}$, such that $c_{v}=0$ for all but finitely many $v$, and for all $v \notin S$,

$$
h_{D, v}(P) \leq c_{v}
$$

for all $P \in R$. This is well-defined, independent of how we write $X \backslash D$ [Vojta 1987, Corollary 1.4.2, Theorem 1.4.11]. There are other essentially equivalent definitions of integrality (see, for example, [ibid., Proposition 1.4.7]), but since our main tools involve heights, this will be the most natural definition for our purposes. 
Let $Z$ be a closed subset of $\mathbb{P}^{n}$ defined over $k$. Let $S$ be a finite set of places of $k$ containing the archimedean places. In this case there is a natural set of integral points on $\mathbb{P}^{n} \backslash Z$. We define $\left(\mathbb{P}^{n} \backslash Z\right)\left(\mathbb{O}_{k, S}\right)$ to be the set of points $P \in \mathbb{P}^{n}(k)$ such that the Zariski closures of $P$ and $Z$ in $\mathbb{P}_{\mathscr{O}_{k}}^{n}$ do not meet over any $v \notin S$. Equivalently, if $D$ is an effective divisor on $\mathbb{P}^{n}$, using the local height functions defined in (1) one easily finds that

$$
\begin{aligned}
\left(\mathbb{P}^{n} \backslash D\right)\left(\mathscr{O}_{k, S}\right) & =\left\{P \in \mathbb{P}^{n}(k) \backslash D: h_{D, v}(P)=0, \forall v \in M_{k} \backslash S\right\} \\
& =\left\{P \in \mathbb{P}^{n}(k) \backslash D: \sum_{v \in S} h_{D, v}(P)=(\operatorname{deg} D) h(P)\right\} .
\end{aligned}
$$

\section{General results}

For the purpose of clarifying our later proofs, we first collect together various elementary facts about heights.

3.1. Heights. Throughout, we let $X$ be a nonsingular projective variety defined over a number field $k$. We first recall the Northcott property for heights associated to ample divisors.

Lemma 8. Let $D$ be an ample divisor on $X$ and $c \in \mathbb{R}$. Then the set of points $\left\{P \in X(k): h_{D}(P)<c\right\}$ is finite.

More generally, finiteness holds for points of $X(\bar{k})$ of bounded degree and bounded ample height. Every height is bounded by a multiple of an ample height [Vojta 1987, Proposition 1.2.9(f)].

Lemma 9. Let $A$ and $D$ be divisors on $X$ with $A$ ample. Then there exists a positive integer $N$ such that

$$
h_{D}(P)<N h_{A}(P)+O(1)
$$

for all $P \in X(\bar{k})$.

The next two lemmas give relations between the height of a point and its image under a rational map.

Lemma 10. Let $\phi \in k(X)$ and let $P_{1}, \ldots, P_{q} \in X(k) \backslash$ Supp $\phi$. Let $S$ be a finite set of places of $k$. Then

$$
\sum_{i=1}^{q} \sum_{v \in S} h_{P_{i}, v}(P)<\sum_{i=1}^{q} \sum_{v \in S} h_{\phi\left(P_{i}\right), v}(\phi(P))+O(1)
$$

for all $P \in X(k) \backslash$ Supp $\phi$ such that $\phi(P) \neq \phi\left(P_{i}\right), i=1, \ldots, q$.

Proof. For an appropriate blow-up $\pi: \tilde{X} \rightarrow X$, where $\pi$ is an isomorphism on $\pi^{-1}(X \backslash \operatorname{Supp} \phi), \phi$ extends to a morphism $\tilde{\phi}: \tilde{X} \rightarrow \mathbb{P}^{1}$ such that $\tilde{\phi}=\phi \circ \pi$ 
on $\pi^{-1}(X \backslash \operatorname{Supp} \phi)$. For a point $P \in X(k) \backslash \operatorname{Supp} \phi$, we let $\tilde{P}=\pi^{-1}(P)$. Let $P \in X(k) \backslash \operatorname{Supp} \phi$ be such that $\phi(P) \neq \phi\left(P_{i}\right), i=1, \ldots, q$. By functoriality of heights,

$$
\sum_{i=1}^{q} \sum_{v \in S} h_{\tilde{\phi}^{*} \phi\left(P_{i}\right), v}(\tilde{P})=\sum_{i=1}^{q} \sum_{v \in S} h_{\phi\left(P_{i}\right), v}(\tilde{\phi}(\tilde{P}))+O(1) .
$$

Since $\tilde{P}_{i}$ is in the support of $\tilde{\phi}^{*} \phi\left(P_{i}\right)$, we have

$$
\sum_{i=1}^{q} \sum_{v \in S} h_{\tilde{P}_{i}, v}(\tilde{P})<\sum_{i=1}^{q} \sum_{v \in S} h_{\tilde{\phi}^{*} \phi\left(P_{i}\right), v}(\tilde{P})+O(1) .
$$

Now the lemma follows from the above two equations, noting that $\tilde{\phi}(\tilde{P})=\phi(P)$ and by functoriality, $h_{\tilde{P}_{i}, v}(\tilde{P})=h_{\pi^{*} P_{i}, v}(\tilde{P})=h_{P_{i}, v}(P)+O(1)$.

Lemma 11. Let $D$ be an effective divisor on $X$ and let $\phi \in k(X)$ be a rational function with every pole contained in Supp $D$. Then for some constant $c>0$,

$$
h(\phi(P))<c h_{D}(P)+O(1)
$$

for all $P \in X(\bar{k}) \backslash \operatorname{Supp} \phi$.

Proof. We use the same notation as in the proof of Lemma 10. Let $P \in X(\bar{k}) \backslash$ Supp $\phi$. By functoriality,

$$
h(\phi(P))=h(\tilde{\phi}(\tilde{P}))=h_{\tilde{\phi}^{*} \infty}(\tilde{P})+O(1) .
$$

Since Supp $\tilde{\phi}^{*} \infty \subset \operatorname{Supp} \pi^{*} D$, there exists a constant $c>0$ such that

$$
h_{\tilde{\phi}^{*} \infty}(\tilde{P})<c h_{\pi^{*} D}(\tilde{P})+O(1) .
$$

By functoriality again, $h_{\pi^{*} D}(\tilde{P})=h_{D}(P)+O(1)$ and the result follows.

The next lemma is crucial in our later proofs.

Lemma 12. Let $E_{1}, \ldots, E_{m}$ be effective divisors on $X$, defined over $k$, such that $\bigcap_{i=1}^{m} E_{i}$ consists of a finite number of points, all defined over $k$. Let $v \in M_{k}$. Then there exists a positive integer $N$ such that

$$
\min _{i} h_{E_{i}, v}(P) \leq N \sum_{Q \in \bigcap_{i=1}^{m} E_{i}(k)} h_{Q, v}(P)+O(1)
$$

for all $P \in X(k) \backslash \bigcup_{i} E_{i}$.

Proof. If $\bigcap_{j=1}^{m} \operatorname{Supp} E_{i}=\varnothing$, then in fact

$$
\min \left\{h_{D_{i_{1}}, v}(P), \ldots, h_{D_{i_{m}}, v}(P)\right\} \leq c
$$


for some constant $c$. This is well known and follows, for instance, from formal properties of heights since in this case $\min \left\{h_{D_{i_{1}}, v}, \ldots, h_{D_{i_{m}}, v}\right\}$ is a local height associated to the trivial divisor.

Otherwise, let $N$ be a positive integer such that $\bigcap_{i=1}^{m} E_{i} \subset N \sum_{Q \in \bigcap_{i=1}^{m} E_{i}(k)} Q$. Then by properties of heights,

$$
\min _{i} h_{E_{i}, v}(P)=h_{\bigcap_{i=1}^{m} E_{i}, v}(P)+O(1) \leq N \sum_{Q \in \bigcap_{i=1}^{m} E_{i}(k)} h_{Q, v}(P)+O(1)
$$

for all $P \in X(k) \backslash \bigcup_{i} E_{i}$.

Finally, we record two basic facts about integral points that follow from the definitions and basic properties of height functions (see also [Vojta 1987, Lemma 1.4.6]).

Lemma 13. Let $D_{1}, \ldots, D_{n}$ be effective divisors on $X$, defined over $k$, and let $D=\sum_{i=1}^{n} D_{i}$. Let $S$ be a finite set of places of $k$ containing the archimedean places and let $R$ be a set of $S$-integral points on $X \backslash D$. Then

$$
\sum_{v \in S} h_{D_{i}, v}(P)=h_{D_{i}}(P)+O(1), \quad i=1, \ldots, n,
$$

for all $P \in R$. If $\phi \in k(X)$ with $\operatorname{Supp} \phi \subset \operatorname{Supp} D$, then there exists a finite set of places $T$ of $k$ such that

$$
\phi(P) \in \mathcal{O}_{k, T}^{*}
$$

for all $P \in R$.

3.2. Results. Let $X$ be a nonsingular projective variety defined over a number field $k$. Let $D_{1}, \ldots, D_{n}$ be effective ample divisors on $X$, defined over $k$, and set $D=\sum_{i=1}^{n} D_{i}$. Let $S$ be a finite set of places of $k$ containing the archimedean places and $R$ a set of $S$-integral points on $X \backslash D$. We need a hypothesis asserting that one can effectively compute the height relations of the last section. We say that $X, D_{1}, \ldots, D_{n}, D, R, S, k$ satisfy $(*)$ if there are height functions associated to $D_{1}, \ldots, D_{n}, D$ and points of $X$ such that:

(1) The finite set in Lemma 8 is effectively computable for $D$ and any $c \in \mathbb{R}$.

(2) The positive integer $N$ and $O(1)$ in Lemma 9 are effectively computable for $D$ and $A=D_{i}, i=1, \ldots, n$.

(3) The $O(1)$ in Lemma 10 is effectively computable for $S$, any $\phi \in k(X)$ with $\operatorname{Supp} \phi \subset \operatorname{Supp} D$, and any set of points $\left\{P_{1}, \ldots, P_{q}\right\} \subset X(k) \backslash \operatorname{Supp} \phi$.

(4) The $O(1)$ in Lemma 11 is effectively computable for $D$ and any $\phi$.

(5) The positive integer $N$ and $O(1)$ in Lemma 12 are effectively computable for any $v \in S$ and subset $\left\{E_{1}, \ldots, E_{m}\right\} \subset\left\{D_{1}, \ldots, D_{n}\right\}$. 
(6) The finite set $T$ and $O(1)$ in Lemma 13 are effectively computable for $R$, $D_{1}, \ldots, D_{n}, D$, and any $\phi$.

(7) The above remain true upon replacing $k$ by a finite extension of $k$ and $S$ by any finite set of places containing the set of places lying above places of $S$.

Additionally, we assume that we can compute in $\operatorname{Pic}(X)$ as follows:

(8) All of the relations between the images of $D_{1}, \ldots, D_{n}$ in $\operatorname{Pic}(X)$ are effectively computable, and for any principal divisor $E$ supported on $D_{1}, \ldots, D_{n}$ one can effectively compute a rational function $\phi \in k(X)$ with $\operatorname{div}(\phi)=E$.

Examples of varieties where (*) is satisfied (for any reasonably defined $R$ ) include curves, projective space, and more generally projective subvarieties of $\mathbb{P}^{N}$ where the divisors $D_{i}$ are hypersurface sections. We explicitly work out the case $X=\mathbb{P}^{2}$ in Section 7. For curves, key algorithms include the computation of Riemann-Roch spaces [Schmidt 1991] and relations amongst points in the Jacobian [Masser 1988].

The main result of this section is a slightly more explicit version of Theorem 1 .

Theorem 14. Let $X$ be a nonsingular projective variety defined over a number field $k$. Let $D_{1}, \ldots, D_{n}$ be effective ample divisors on $X$ defined over $k$. Let $D=\sum_{i=1}^{n} D_{i}$. Let $m \leq n$ be a positive integer such that for all subsets $I \subset\{1, \ldots, n\}$, $|I|=m$, the set $\bigcap_{i \in I}\left(\operatorname{Supp} D_{i}\right)(\bar{k})$ consists of finitely many points. Suppose that for each point $P \in(\operatorname{Supp} D)(\bar{k})$, there exists a nonconstant rational function $\phi \in k(X)$ satisfying $P \notin \operatorname{Supp} \phi$ and $\operatorname{Supp} \phi \subset \operatorname{Supp} D$. Let $S$ be a set of places of $k$ containing the archimedean places with

$$
(m-1)|S|<n .
$$

Let $R$ be a set of $S$-integral points on $X \backslash D$. Suppose that $X, D_{1}, \ldots, D_{n}, D, R, S, k$ satisfy (*) in Section 3.2. Then $R$ is contained in an effectively computable proper closed subset $Z$ of $X$. Explicitly, for a point $P \in X(\bar{k})$, let

$$
\Phi_{P}=\left\{\phi \in k(X)^{*}: P \notin \operatorname{Supp} \phi, \operatorname{Supp} \phi \subset \operatorname{Supp} D\right\}
$$

and let

$$
T=\bigcup_{\substack{I \subset\{1, \ldots, n\} \\|I|=m}} \bigcap_{i \in I}\left(\operatorname{Supp} D_{i}\right)(\bar{k}) .
$$

Then we may take $Z$ to consist of a finite effectively computable set of points together with the Zariski closure of the set

$$
\bigcup_{P \in T} \bigcap_{\phi \in \Phi_{P}}\{Q \in X(\bar{k}): \phi(Q)=\phi(P)\} .
$$


Remark 15. As is typical, more generally one could replace ample with big in the theorem by modifying the theorem slightly (e.g., increasing the exceptional set $Z$ to account for the base loci of certain divisors).

If $D_{1}, \ldots, D_{r+1}$ are nontrivial effective divisors on a variety $X$ that generate a subgroup of $\operatorname{Pic}(X)$ of rank $r$ and pairwise do not have any common components, then there exists a nonconstant rational function $\phi$ on $X$ with all zeros and poles contained in the support of $\sum_{i=1}^{r+1} D_{i}$. Using this fact to construct appropriate rational functions $\phi$ in Theorem 14, we immediately obtain the following corollary.

Corollary 16. Let $X$ be a nonsingular projective variety defined over a number field $k$. Let $D_{1}, \ldots, D_{n}$ be ample effective divisors on $X$, defined over $k$, that generate a subgroup of $\operatorname{Pic}(X)$ of rank $r$ and pairwise do not have any common components. Let $D=\sum_{i=1}^{n} D_{i}$. Let $m \leq n$ be a positive integer such that for all subsets $I \subset\{1, \ldots, n\},|I|=m$, the set $\bigcap_{i \in I}\left(\operatorname{Supp} D_{i}\right)(\bar{k})$ consists of finitely many points. Suppose that the intersection of the supports of any $n-r$ of the divisors $D_{i}$ is empty. Let $S$ be a set of places of $k$ containing the archimedean places with

$$
(m-1)|S|<n \text {. }
$$

Let $R$ be a set of $S$-integral points on $X \backslash D$. Suppose that $X, D_{1}, \ldots, D_{n}, D, R, S, k$ satisfy (*). Then $R$ is contained in an effectively computable proper closed subset $Z$ of $X$.

Of particular interest is the case where $X$ is a surface.

Corollary 17. Let $X$ be a nonsingular projective surface over a number field $k$. Let $D_{1}, \ldots, D_{n}$ be ample effective divisors on $X$, defined over $k$, that generate a subgroup of $\operatorname{Pic}(X)$ of rank $r$ and pairwise do not have any common components. Suppose that the intersection of the supports of any $n-r$ of the divisors $D_{i}$ is empty. Let $S$ be a set of places of $k$ containing the archimedean places with

$$
|S|<n \text {. }
$$

Let $R$ be a set of $S$-integral points on $X \backslash D$. Suppose that $X, D_{1}, \ldots, D_{n}, D, R, S, k$ satisfy (*). Then $R$ is contained in an effectively computable proper closed subset $Z$ of $X$. Let

$$
T=\bigcup_{i \neq j}\left(D_{i} \cap D_{j}\right)(\bar{k}),
$$

and let $\Phi_{P}$ be as in Theorem 14. Then we may take $Z$ to consist of a finite effectively computable set of points together with the Zariski closure of the set

$$
\bigcup_{P \in T} \bigcap_{\phi \in \Phi_{P}}\{Q \in X(\bar{k}): \phi(Q)=\phi(P)\} .
$$


3.3. Proofs. The key tool in this section is the main theorem from the theory of linear forms in logarithms, which we now state in the language of heights (see Theorem 24 for a completely explicit version).

Theorem 18. Let $k$ be a number field and $S$ a finite set of places of $k$ containing the archimedean places. Let $v \in M_{k}, \alpha \in k^{*}$, and $\epsilon>0$. Then there exists an effective constant $C$ such that

$$
h_{\alpha, v}(x) \leq \epsilon h(x)+C
$$

for all $x \in 0_{k, S}^{*}, x \neq \alpha$.

We note that with an ineffective constant $C$, the theorem follows easily from Roth's theorem. Before proving Theorem 14, we prove a result which can be regarded as a higher-dimensional version of Theorem 18.

Theorem 19. Let $X$ be a nonsingular projective variety defined over a number field $k$ and let $D$ be an effective divisor on $X$ defined over $k$. Let $\phi \in k(X)$ be a nonconstant rational function with $\operatorname{Supp} \phi \subset \operatorname{Supp} D$. Let $S$ be a finite set of places of $k$ and $R$ a set of $S$-integral points on $X \backslash D$. Suppose that $X, D, R, S, k$ satisfy (3), (4), (6), (7) of Section 3.2. Let $P_{1}, \ldots, P_{q} \in X(k) \backslash \operatorname{Supp} \phi$ and $\epsilon>0$. Then

$$
\sum_{i=1}^{q} \sum_{v \in S} h_{P_{i}, v}(P)<\epsilon h_{D}(P)+O(1)
$$

for all $P \in R \backslash Z$, where $Z$ is the proper closed subset of $X$ defined as the Zariski closure of the set

$$
\left\{P \in X(\bar{k}): \phi(P)=\phi\left(P_{i}\right) \text { for some } i \in\{1, \ldots, q\}\right\} .
$$

Here, as well as elsewhere, the implicit constant in the $O(1)$ is an effective constant.

Proof. By Lemma 13, since $R$ is a set of $S$-integral points on $X \backslash D$, without loss of generality, after enlarging $S$ we can assume that $\phi(P) \in \mathcal{O}_{k, S}^{*}$ for all $P \in R$. Then by Theorem 18,

$$
\sum_{i=1}^{q} \sum_{v \in S} h_{\phi\left(P_{i}\right), v}(\phi(P))<\epsilon h(\phi(P))+O(1)
$$

for all $P \in R \backslash Z$. By Lemma 10,

$$
\sum_{i=1}^{q} \sum_{v \in S} h_{P_{i}, v}(P)<\sum_{i=1}^{q} \sum_{v \in S} h_{\phi\left(P_{i}\right), v}(\phi(P))+O(1)
$$

for all $P \in X(k) \backslash(Z \cup \operatorname{Supp} \phi)$. By Lemma 11,

$$
\epsilon h(\phi(P))<\epsilon \operatorname{ch}_{D}(P)+O(1)
$$


for some positive constant $c$ and all $P \in X(k) \backslash$ Supp $D$. Replacing $\epsilon$ by $\epsilon / c$ and combining the above inequalities yields

$$
\sum_{i=1}^{q} \sum_{v \in S} h_{P_{i}, v}(P)<\epsilon h_{D}(P)+O(1)
$$

for all $P \in R \backslash Z$.

We now prove Theorem 14.

Proof of Theorem 14. By Lemma 13, since $R$ is a set of $S$-integral points on $X \backslash D$, we have

$$
\sum_{v \in S} h_{D_{i}, v}(P)=h_{D_{i}}(P)+O(1), \quad i=1, \ldots, n,
$$

for all $P \in R$. Let $P \in R$. Then for each $i$, there exists a place $v \in S$ such that $h_{D_{i}, v}(P) \geq(1 /|S|) h_{D_{i}}(P)+O(1)$. Since $(m-1)|S|<n$, there exists a place $v \in S$ and distinct elements $i_{1}, i_{2}, \ldots, i_{m} \in\{1, \ldots, n\}$ such that

$$
\min \left\{h_{D_{i_{1}}, v}(P), \ldots, h_{D_{i_{m}}, v}(P)\right\} \geq \frac{1}{|S|} \min _{j} h_{D_{i_{j}}}(P)+O(1) .
$$

By Lemma 9, there exists a positive integer $N$ such that

$$
h_{D}(P) \leq N h_{D_{i}}(P)+O(1)
$$

for all $i$ and all $P \in X(\bar{k})$. So for $P \in R$,

$$
\min \left\{h_{D_{i_{1}}, v}(P), \ldots, h_{D_{i_{m}}, v}(P)\right\} \geq \frac{1}{N|S|} h_{D}(P)+O(1) .
$$

The theorem is then a consequence of the following lemma.

Lemma 20. Let $m, X, D_{1}, \ldots, D_{n}, R, S, k$ be as in the hypotheses of Theorem 14 . Let $\epsilon>0, v \in S$, and let $i_{1}, \ldots, i_{m} \in\{1, \ldots, n\}$ be distinct integers. Then the set of points

$$
\left\{P \in R: \min \left\{h_{D_{i_{1}}, v}(P), \ldots, h_{D_{i_{m}}, v}(P)\right\}>\epsilon h_{D}(P)\right\}
$$

is contained in an effectively computable proper closed subset $Z$ of $X$. For $P \in X(\bar{k})$, let $\Phi_{P}$ be the set from Theorem 14 and let

$$
T=\bigcap_{j=1}^{m}\left(\operatorname{Supp} D_{i_{j}}\right)(\bar{k}) .
$$

Then we may take $Z$ to consist of a finite effectively computable set of points together with the Zariski closure of the set

$$
\bigcup_{P \in T} \bigcap_{\phi \in \Phi_{P}}\{Q \in X(\bar{k}): \phi(Q)=\phi(P)\} .
$$


Proof. If $L$ is a finite extension of $k$ and $w$ is a place of $L$ lying above $v$, then we can define a local height function

$$
h_{D_{i}, w}(P)=\frac{\left[L_{w}: k_{v}\right]}{[L: k]} h_{D_{i}, v}(P)
$$

for all $P \in X(k) \backslash D_{i}$. It follows that without loss of generality, after replacing $k$ by a finite extension of $k$ and $v$ by a place lying above $v$, we may assume that every point $P \in X(\bar{k})$ in the intersection $\bigcap_{j=1}^{m}$ Supp $D_{i_{j}}$ is defined over $k$ (note that by hypothesis this intersection consists of a finite number of points).

If $\bigcap_{j=1}^{m} \operatorname{Supp} D_{i_{j}}=\varnothing$, then by Lemma 12,

$$
\min \left\{h_{D_{i_{1}}, v}(P), \ldots, h_{D_{i_{m}}, v}(P)\right\} \leq C
$$

for some effective constant $C$. In this case, the lemma follows immediately from the fact that since $D$ is ample, the set of points $\left\{P \in X(k): h_{D}(P)<C / \epsilon\right\}$ is finite.

Suppose now that $\bigcap_{j=1}^{m} \operatorname{Supp} D_{i_{j}} \neq \varnothing$, in which case it consists of a finite number $q$ of points. By Lemma 12, there exists a positive integer $N$ such that

for all $P \in X(k) \backslash \bigcup_{j} D_{i_{j}}$.

$$
\min _{j} h_{D_{i_{j}}, v}(P) \leq N \sum_{Q \in \bigcap_{j=1}^{m} D_{i_{j}}(k)} h_{Q, v}(P)+O(1)
$$

Let $Q \in \bigcap_{j=1}^{m} D_{i_{j}}(k)$. Note that $\Phi_{Q}$ is a monoid under multiplication, generated by $k^{*}$ and finitely many rational functions in $k(X)^{*}$. Let $\epsilon>0$. Since $R$ is a set of $S$-integral points on $X \backslash D$, applying Theorem 19 multiple times yields the inequality

$$
h_{Q, v}(P)<\frac{\epsilon}{2 N q} h_{D}(P)+O(1)
$$

for all $P \in R \backslash Z_{Q}$, where $Z_{Q}$ is the Zariski closure of the set

$$
\bigcap_{\phi \in \Phi_{Q}}\{P \in X(\bar{k}): \phi(P)=\phi(Q)\} .
$$

Summing over all points in $\bigcap_{j=1}^{m} D_{i_{j}}(k)$, we obtain

$$
\min _{j} h_{D_{i_{j}}, v}(P) \leq N \sum_{Q \in \bigcap_{j=1}^{m} D_{i_{j}}(k)} h_{Q, v}(P)+O(1)<\frac{\epsilon}{2} h_{D}(P)+C
$$

for all $P \in R \backslash Z$, where

$$
Z=\bigcup_{Q \in \bigcap_{j=1}^{m} D_{i_{j}}(k)} Z_{Q}
$$

and $C$ is an effectively computable constant. So if $P \in R \backslash Z$ satisfies

$$
\min _{j} h_{D_{i_{j}}, v}(P)>\epsilon h_{D}(P),
$$


then $h_{D}(P)<\frac{2}{\epsilon} C$. It follows that

$$
\left\{P \in R: \min _{j} h_{D_{i_{j}}, v}(P)>\epsilon h_{D}(P)\right\} \subset Z \cup\left\{P \in X(k): h_{D}(P)<\frac{2}{\epsilon} C\right\},
$$

where $Z$ is a proper Zariski closed subset of $X$ and the last set on the right is finite.

\section{An application to polynomial unit equations}

We prove a complete version of Corollary 4 from the introduction.

Corollary 21. Let $f \in k[x, y]$ be a polynomial of degree $d$ such that $f(0,0)=$ $c_{0} \neq 0$ and $x^{d}$ and $y^{d}$ have nonzero coefficients $c_{x}$ and $c_{y}$ in $f$, respectively. Let $S$ be a set of places of $k$ containing the archimedean places with $|S| \leq 3$. Then the set of solutions to

$$
f(u, v)=w, \quad u, v, w \in \mathbb{O}_{k, S}^{*},
$$

consists of a finite effectively computable set and a finite number of infinite families of solutions where one of $u, v$, or $w$ is constant. Let

$$
\begin{aligned}
& T_{1}=\left\{a \in \mathcal{O}_{k, S}^{*}:(x-a) \mid\left(f(x, y)-c_{y} y^{d}\right), c_{y} \in \mathcal{O}_{k, S}^{*}\right\}, \\
& T_{2}=\left\{a \in \mathcal{O}_{k, S}^{*}:(y-a) \mid\left(f(x, y)-c_{x} x^{d}\right), c_{x} \in \mathcal{O}_{k, S}^{*}\right\}, \\
& T_{3}=\left\{a \in \mathcal{O}_{k, S}^{*}:(y-a x) \mid\left(f(x, y)-c_{0}\right), c_{0} \in \mathcal{O}_{k, S}^{*}\right\} .
\end{aligned}
$$

Then the infinite families of solutions are

$$
\begin{array}{lll}
(u, v, w)=\left(a, t, c_{y} t^{d}\right), & t \in \mathcal{O}_{k, S}^{*}, & \text { for each } a \in T_{1}, \\
(u, v, w)=\left(t, a, c_{x} t^{d}\right), & t \in \mathcal{O}_{k, S}^{*}, & \text { for each } a \in T_{2}, \\
(u, v, w)=\left(t, a t, c_{0}\right), & t \in \mathcal{O}_{k, S}^{*}, & \text { for each } a \in T_{3} .
\end{array}
$$

Proof. It will be convenient to work with the homogenized polynomial

$$
F(x, y, z)=z^{d} f(x / z, y / z) .
$$

Consider $\mathbb{P}^{2}$ with homogeneous coordinates $(x, y, z)$ and let $D_{1}, D_{2}, D_{3}, D_{4}$ be the curves defined by $x=0, y=0, z=0$, and $F(x, y, z)=0$, respectively. Let $D=\sum_{i=1}^{4} D_{i}$. Let

$$
R=\left\{(u, v, 1) \in \mathbb{P}^{2}(k): u, v, f(u, v) \in \mathbb{O}_{k, S}^{*}\right\} .
$$

Then $R \subset\left(\mathbb{P}^{2} \backslash D\right)\left(\mathscr{O}_{k, S}\right)$. Let $\{i, j, k\}=\{1,2,3\}$ and $P \in\left(D_{i} \cap D_{4}\right)(\bar{k})$. Then, using the notation of Corollary 17, the Zariski closure of $\bigcap_{\phi \in \Phi_{P}}\{Q \in X(\bar{k}): \phi(Q)=\phi(P)\}$ is a line through $P$ and the unique point of $D_{j} \cap D_{k}$. Now let $P_{1}=(1,0,0)$, 
$P_{2}=(0,1,0)$, and $P_{3}=(0,0,1)$, so that $\left\{P_{i}\right\}=\bigcap_{j \in\{1,2,3\} \backslash\{i\}} D_{j}(\bar{k})$. Let $Z_{i}$ be the Zariski closure of

$$
\bigcap_{\phi \in \Phi_{P_{i}}}\left\{Q \in X(\bar{k}): \phi(Q)=\phi\left(P_{i}\right)\right\}
$$

for $i=1,2,3$. Since $\left(F(x, y, z) / x^{d}\right)\left(P_{1}\right)=c_{x},\left(F(x, y, z) / y^{d}\right)\left(P_{2}\right)=c_{y}$, and $\left(F(x, y, z) / z^{d}\right)\left(P_{3}\right)=c_{0}$, it follows that we have the equations

$$
Z_{1}: F(x, y, z)=c_{x} x^{d}, \quad Z_{2}: F(x, y, z)=c_{y} y^{d}, \quad Z_{3}: F(x, y, z)=c_{0} z^{d} .
$$

Let $Z$ be the closed subset of $\mathbb{P}^{2}$ consisting of all lines connecting points of $\left(D_{i} \cap D_{j}\right)(\bar{k})$ with points of $\left(D_{k} \cap D_{l}\right)(\bar{k})$, where $\{i, j, k, l\}=\{1,2,3,4\}$, together with the closed subsets $Z_{1}, Z_{2}$, and $Z_{3}$. Then it follows from Corollary 17 that $R \backslash Z$ consists of a finite effectively computable set of points (in fact, an explicit height bound for points in this set follows from Theorem 7).

Now let $C$ be a geometrically irreducible curve in $Z$. If $C$ is not defined over $k$ and $C^{\prime}$ is any nontrivial conjugate of $C$ over $k$, then $C(k) \subset\left(C \cap C^{\prime}\right)(\bar{k})$, a finite effectively computable set. In particular, $R \cap C$ is finite and effectively computable. Assume now that $C$ is defined over $k$. Then $R \cap C$ is a set of integral points on $C \backslash(C \cap D)$. Consider the rational functions on $C$ given by $\phi_{1}=\left.(x / z)\right|_{C}$ and $\phi_{2}=\left.(y / z)\right|_{C}$. The functions $\phi_{1}$ and $\phi_{2}$ have zeros and poles only in $C \cap D$. If $\phi_{1}$ and $\phi_{2}$ are multiplicatively independent modulo $k^{*}$, then Bilu's Theorem 2 implies that $R \cap C$ is finite and effectively computable. Suppose now that this is not the case. Then this easily implies that $C$ is given by an equation $x^{m} y^{n-m}=a z^{n}$, $x^{m} z^{n-m}=a y^{n}$, or $y^{m} z^{n-m}=a x^{n}$ for some nonnegative integers $m$ and $n$ and $a \in k^{*}$. Suppose first that $n \geq 2$. Then $C$ is a component of $Z_{1}, Z_{2}$, or $Z_{3}$. Suppose that, say, $C$ is given by $x^{m} y^{n-m}=a z^{n}$ and is a component of $Z_{1}$. Then $F(x, y, z)=c_{x} x^{d}+\left(x^{m} y^{n-m}-a z^{n}\right) g(x, y, z)$ for some homogeneous polynomial $g(x, y, z) \in k[x, y, z]$. Since $C$ is geometrically irreducible and $n \geq 2$, we must have $0<m<n$. But from the form of $F(x, y, z)$ we then see that $y^{d}$ cannot have a nonzero coefficient in $F(x, y, z)$, contradicting our assumptions. The other possible cases are similar and we conclude that $n=1$. So $C$ is defined by a linear form $x-a z, y-a z$, or $y-a x$, for some $a \in k^{*}$.

Suppose that $C$ is defined by $x-a z=0$. If $R \cap C \neq \varnothing$ then $a \in 0_{k, S}^{*}$, which we now assume. Since $y^{d}$ must have a nonzero coefficient in $F(x, y, z)$, it follows that $C$ cannot be an irreducible component of $Z_{1}$ or $Z_{3}$. If $C$ is an irreducible component of $Z_{2}$, then $f(x, y)=c_{y} y^{d}+(x-a) g(x, y)$ for some polynomial $g(x, y) \in k[x, y]$. If $C$ connects a point of $D_{i} \cap D_{j}$ with a point of $D_{k} \cap D_{l}$, where $\{i, j, k, l\}=\{1,2,3,4\}$, then it must be that $C$ connects the unique point of $D_{1} \cap D_{3}$ with a point of $D_{2} \cap D_{4}$. If $C$ intersects $D$ in more than two points over $\bar{k}$, then it follows easily again from Theorem 2 that $R \cap C$ is finite and effectively computable. So suppose that 
$|(C \cap D)(\bar{k})| \leq 2$, in which case $|(C \cap D)(\bar{k})|=2$. Then the fact that $C \cap D_{4}$ consists of a single point contained in $D_{2}$ implies that $f(x, y)=c_{y} y^{d}+(x-a) g(x, y)$ for some polynomial $g(x, y) \in k[x, y]$. So in any case, $f(x, y)=c_{y} y^{d}+(x-a) g(x, y)$ for some polynomial $g(x, y) \in k[x, y]$. Now $C \cap R \neq \varnothing$ implies that $c_{y} \in \mathbb{O}_{k, S}^{*}$, and in this case we find that $C \cap R=\left\{(a, t, 1): t \in \mathbb{O}_{k, S}^{*}\right\}$, leading to the infinite family of solutions $(u, v, w)=\left(a, t, c_{y} t^{d}\right)$, where $t \in \mathbb{O}_{k, S}^{*}$.

The cases where $C$ is defined by $y-a z$ or $y-a x$ are similar, and we are led to the classification of the infinite families in the theorem.

\section{Comparison with Runge's method}

An old method of Runge yields effective finiteness for the set of integral points on certain curves. In its most basic form, Runge proved:

Theorem 22 [Runge 1887]. Let $f \in \mathbb{Q}[x, y]$ be an absolutely irreducible polynomial of total degree $n$. Let $f_{0}$ denote the leading form of $f$, that is, the sum of the terms of total degree $n$ in $f$. Suppose that $f_{0}$ factors as $f_{0}=g_{0} h_{0}$, where $g_{0}, h_{0} \in \mathbb{Q}[x, y]$ are nonconstant relatively prime polynomials. Then the set of solutions to

$$
f(x, y)=0, \quad x, y \in \mathbb{Z},
$$

is finite and effectively computable.

We will state a general higher-dimensional version of Runge's method from [Levin 2008] (see [Bombieri 1983] for earlier work on curves). Before stating a higher-dimensional version, we give some definitions which allow for varying sets of places and number fields. It will be more convenient here to use a definition of integrality involving regular functions. Let $V$ be a variety (not necessarily projective or affine) defined over a number field $k$. Let $s$ be a positive integer. We call a set $R \subset V(\bar{k})$ a set of $s$-integral points on $V$ if for every point $P \in R$ there exists a set of places $S_{P}$ of $k(P)$, containing the archimedean places of $k(P)$, such that $\left|S_{P}\right| \leq s$ and for every regular function $\phi \in \bar{k}(V)$ on $V$ there exists a nonzero constant $c_{\phi} \in k^{*}$, independent of $P$, such that $\left|c_{\phi} \phi(P)\right|_{v} \leq 1$ for all places $v$ of $k(P)$ not in $S_{P}$ (extending each place $v$ of $k(P)$ to $\bar{k}$ in some fixed way). With these definitions, we have a higher-dimensional version of Runge's theorem:

Theorem 23. Let $X$ be a nonsingular projective variety defined over a number field $k$. Let $D=\sum_{i=1}^{r} D_{i}$ be a divisor on $X$, with $D_{1}, \ldots, D_{r}$ effective divisors defined over $k$. Suppose that the intersection of any $m+1$ of the supports of the divisors $D_{i}$ is empty. Let s be a positive integer satisfying

$$
m s<r .
$$

Let $R$ be a set of s-integral points on $X \backslash D$. Suppose that for every regular function 
$\phi \in \bar{k}(X)$ on $X \backslash D$, the constant $c_{\phi}$ in the definition of s-integral is effectively computable with respect to $R$. Suppose also that one can effectively compute a basis of $L\left(n D_{i}\right)$ for all $n>0$ and all $i$. Then the following statements hold.

(a) If $\kappa\left(D_{i}\right)>0$ for all $i$, then $R$ is contained in an effectively computable proper Zariski closed subset $Z \subset X$.

(b) If $D_{i}$ is big for all $i$, then there exists an effectively computable proper Zariski closed subset $Z \subset X$, independent of $R$, such that the set $R \backslash Z$ is finite (and effectively computable).

(c) If $D_{i}$ is ample for all $i$, then $R$ is finite and effectively computable.

We now briefly discuss some of the advantages and disadvantages of the higherdimensional Runge method as compared to our results here. To begin, in some respects the conditions on the divisors $D_{i}$ in Theorem 23 are weaker than the conditions required in Theorem 14. The divisors in Theorem 23 are not required to be ample or big (though one still needs $\kappa\left(D_{i}\right)>0$ ) and furthermore there is no linear equivalence condition present in Theorem 23. On the other hand, for the necessary rational functions $\phi$ to exist in Theorem 14, it is necessary that the subgroup of the Picard group generated by the divisors $D_{i}$ not be too large (this condition is more explicitly present in Corollary 16). Another advantage of Theorem 23 is that the result is uniform in $|S|$, giving degeneracy of integral points even as $S$ and $k$ vary subject to an appropriate inequality. This is also, however, a limitation of Theorem 23, as many results are simply not true in this generality (e.g., the unit equation $u+v=1$ likely has infinitely many solutions in rational $S$-units, $|S| \leq 3$, since for instance there are expected to be infinitely many Mersenne primes). It is not apparent from the statement of Theorem 23, but when Runge's method applies it also gives much smaller bounds than techniques coming from Baker's theorem.

As compared to Theorem 23, we note that the intersection condition on the divisors $D_{i}$ in Theorem 14 is much weaker, especially on surfaces. For instance, the intersection condition on the divisors in Corollary 17 allows for highly degenerate configurations of the divisors $D_{i}$. Finally, we note that even in cases where the divisors $D_{i}$ are in general position, the crucial inequality involving $|S|$ in Theorem 14 is superior to the inequality in Theorem 23. This is particularly notable in the case of surfaces, where the superior inequality on $|S|$ is crucial, for instance, in proving Corollary 4.

\section{Effective inequalities}

In preparation for the next section, we recall several needed inequalities and prove them here. 
6.1. Linear forms in logarithms. The deepest effective result we need is from the theory of linear forms in logarithms. We give a statement in terms of local heights, based on an inequality of Bérczes, Evertse, and Győry [Bérczes et al. 2009].

Theorem 24. Let $k$ be a number field of degree $\delta$ and let $G$ be a finitely generated multiplicative subgroup of $k^{*}$ of rank $t>0$. Let $\alpha \in k^{*}$ and $v \in M_{k}$. Let $0<\epsilon<1$. Then if $x \in G, x \neq \alpha$, we have

$$
h_{\alpha, v}(x) \leq \epsilon h(x)+c_{1}(\epsilon, k, G, v, \alpha)+\log 2,
$$

where

$$
\begin{aligned}
& c_{1}(\epsilon, k, G, v, \alpha) \\
& \quad=6.4 \frac{c_{2}(\delta, t) N(v)}{\epsilon \log N(v)} Q_{G} \max \{h(\alpha), 1\} \max \left\{\log \frac{c_{2}(\delta, t) N(v)}{\epsilon}, \log ^{*} Q_{G}\right\}, \\
& c_{2}(\delta, t)=36(16 e \delta)^{3 t+5}\left(\log ^{*} \delta\right)^{2} .
\end{aligned}
$$

Here, if $G$ is a finitely generated multiplicative subgroup of $\overline{\mathbb{Q}}$ of rank $t>0$, then we let $Q_{G}$ be the minimum value of

$$
h\left(u_{1}\right) \cdots h\left(u_{t}\right),
$$

where $u_{1}, \ldots, u_{t}$ are generators for $G$ modulo the roots of unity in $G$. We let $Q_{S}=Q_{\bigcirc_{k, S}^{*}}$.

Proof. Let $x \in G, x \neq \alpha$. Suppose first that

$$
h_{\alpha, v}(x) \leq \epsilon h(x)+h_{v}(\alpha)+h_{v}(1 / \alpha)+h_{v}(2) .
$$

Then, using that $h(\alpha)=h(1 / \alpha)$, we have

$$
h_{\alpha, v}(x) \leq \epsilon h(x)+2 h(\alpha)+\log 2 \leq \epsilon h(x)+c_{1}(\epsilon, k, G, v, \alpha)+\log 2,
$$

as $2 h(\alpha)$ is easily bounded by $c_{1}(\epsilon, k, G, v, \alpha)$. Suppose now that

$$
h_{\alpha, v}(x)>\epsilon h(x)+h_{v}(\alpha)+h_{v}(1 / \alpha)+h_{v}(2) .
$$

We have

$$
\begin{aligned}
h_{\alpha, v}(x) & =\log \frac{\max \left\{\|\alpha\|_{v}, 1\right\} \max \left\{\|x\|_{v}, 1\right\}}{\|x-\alpha\|_{v}} \\
& =\log \frac{\max \left\{\|\alpha\|_{v}, 1\right\} \max \left\{\left\|\frac{x}{\alpha}\right\|_{v},\left\|\frac{1}{\alpha}\right\|_{v}\right\}}{\left\|\frac{x}{\alpha}-1\right\|_{v}} .
\end{aligned}
$$

Now

$$
\left\|\frac{x}{\alpha}\right\|_{v}=\left\|\left(\frac{x}{\alpha}-1\right)+1\right\|_{v} \leq \epsilon_{v}^{\prime}(2) \max \left\{\left\|\frac{x}{\alpha}-1\right\|_{v}, 1\right\} .
$$


It follows that

$$
h_{\alpha, v}(x) \leq \log \frac{\epsilon_{v}^{\prime}(2) \max \left\{\|\alpha\|_{v}, 1\right\} \max \left\{\left\|\frac{x}{\alpha}-1\right\|_{v},\left\|\frac{1}{\alpha}\right\|_{v}, 1\right\}}{\left\|\frac{x}{\alpha}-1\right\|_{v}} .
$$

If

$$
\max \left\{\left\|\frac{x}{\alpha}-1\right\|_{v},\left\|\frac{1}{\alpha}\right\|_{v}, 1\right\}=\left\|\frac{x}{\alpha}-1\right\|_{v},
$$

then $h_{\alpha, v}(x) \leq \log \epsilon_{v}^{\prime}(2) \max \left\{\|\alpha\|_{v}, 1\right\} \leq h_{v}(\alpha)+h_{v}(2)$, contradicting our assumptions. Then we must have

$$
\begin{aligned}
h_{\alpha, v}(x) & \leq \log \frac{\epsilon_{v}^{\prime}(2) \max \left\{\|\alpha\|_{v}, 1\right\} \max \left\{\left\|\frac{1}{\alpha}\right\|_{v}, 1\right\}}{\left\|\frac{x}{\alpha}-1\right\|_{v}} \\
& \leq h_{v}(2)+h_{v}(\alpha)+h_{v}(1 / \alpha)-\log \left\|\frac{x}{\alpha}-1\right\|_{v} .
\end{aligned}
$$

So

$$
\log \left\|\frac{x}{\alpha}-1\right\|_{v}<-\epsilon h(x) .
$$

By [Bérczes et al. 2009, Theorem 4.2], this implies that

$$
h(x) \leq c_{1}(\epsilon, k, G, v, \alpha) .
$$

Now we note that by Lemma 32, proved later in this section, for any $x \in k, x \neq \alpha$,

$$
h_{\alpha, v}(x) \leq \log 2+\sum_{v \in M_{k}} h_{\alpha, v}(x)=\log 2+h(x) .
$$

Thus,

$$
h_{\alpha, v}(x) \leq \epsilon h(x)+c_{1}(\epsilon, k, G, v, \alpha)+\log 2 .
$$

6.2. Hilbert's Nullstellensatz. We will need an effective version of Hilbert's Nullstellensatz. We use the following version, due to Masser and Wüstholz [1983].

Theorem 25 (effective Hilbert's Nullstellensatz). Let $k$ be a number field and let $p_{1}, \ldots, p_{m}, q \in \mathbb{O}_{k}\left[x_{1}, \ldots, x_{n}\right]$ be polynomials of degree at most $d \geq 1$ such that $q$ vanishes at all common zeros of $p_{1}, \ldots, p_{m}$ in $\mathbb{A}^{n}(\bar{k})$. Then there exists a positive integer $M \leq(8 d)^{2^{n}}$ and polynomials $a_{1}, \ldots, a_{m} \in \mathbb{O}_{k}\left[x_{1}, \ldots x_{n}\right]$ of degrees at most $(8 d)^{2^{n}+1}$, such that

$$
a q^{M}=a_{1} p_{1}+\cdots+a_{m} p_{m}
$$

for some nonzero element $a \in \mathbb{O}_{k}$. Furthermore, if

$$
h_{\infty}=\log \max _{\substack{v \in M_{k} \\ v \mid \infty}}\left\{\left|p_{1}\right|_{v}, \ldots,\left|p_{m}\right|_{v},|q|_{v}\right\},
$$

then

$$
\log \max _{\substack{v \in M_{k} \\ v \mid \infty}}\left\{\left|a_{1}\right|_{v}, \ldots,\left|a_{m}\right|_{v},|a|_{v}\right\} \leq(8 d)^{2^{n+1}-1}\left(h_{\infty}+8 d \log 8 d\right) .
$$


Remark 26. Applying the theorem appropriately to $\mathbb{A}^{n}$, it's clear that the same result holds for homogeneous polynomials $p_{1}, \ldots, p_{m}, q \in \mathbb{O}_{k}\left[x_{1}, \ldots, x_{n}\right]$ such that $q$ vanishes at all common zeros of $p_{1}, \ldots, p_{m}$ in $\mathbb{P}^{n-1}(\bar{k})$. Furthermore, in this case one can clearly choose $a_{1}, \ldots, a_{m}$ to be homogeneous polynomials with $\operatorname{deg} a_{i}=M \operatorname{deg} q-\operatorname{deg} p_{i}$.

6.3. Arithmetic Bézout. We will make use of the following arithmetic Bézout theorem for curves in $\mathbb{P}^{2}$, which is essentially a special case of a general arithmetic Bézout theorem of Philippon [1995].

Theorem 27. Let $C_{1}$ and $C_{2}$ be distinct curves in $\mathbb{P}^{2}$ over $\overline{\mathbb{Q}}$. Then

$$
\sum_{P \in\left(C_{1} \cap C_{2}\right)(\overline{\mathbb{Q}})} h(P) \leq\left(\operatorname{deg} C_{1}\right) h\left(C_{2}\right)+\left(\operatorname{deg} C_{2}\right) h\left(C_{1}\right)+4\left(\operatorname{deg} C_{1}\right)\left(\operatorname{deg} C_{2}\right) .
$$

Proof. We will denote the height used by Philippon [1995] by $h_{\mathrm{Ph}}$. By [ibid., Proposition 4],

$$
\sum_{P \in\left(C_{1} \cap C_{2}\right)(\overline{\mathbb{Q}})} h_{\mathrm{Ph}}(P) \leq\left(\operatorname{deg} C_{1}\right) h_{\mathrm{Ph}}\left(C_{2}\right)+\left(\operatorname{deg} C_{2}\right) h_{\mathrm{Ph}}\left(C_{1}\right) .
$$

From [ibid., p. 347], for $i=1,2$ we have

$$
h_{\mathrm{Ph}}\left(C_{i}\right)=h_{\mathrm{Ph}}\left(f_{i}\right)+\frac{\operatorname{deg} C_{i}}{2}
$$

and from the definitions of the heights, easy estimates give

$$
h_{\mathrm{Ph}}\left(f_{i}\right) \leq h\left(f_{i}\right)+\left(\log 2+\frac{3}{4}\right) \operatorname{deg} C_{i}=h\left(C_{i}\right)+\left(\log 2+\frac{3}{4}\right) \operatorname{deg} C_{i} .
$$

So

$$
h_{\mathrm{Ph}}\left(C_{i}\right) \leq h\left(C_{i}\right)+2 \operatorname{deg} C_{i} \quad \text { for } i=1,2 .
$$

Finally, we note that if $P$ is a point in $\mathbb{P}^{n}$, then $h_{\mathrm{Ph}}(P)$ is the usual height $h(P)$ except that at the archimedean places one uses the $\ell^{2}$-norm. In particular, $h(P) \leq h_{\mathrm{Ph}}(P)$. Combining the above inequalities gives the result.

6.4. Units and regulators. Let $k$ be a number field of degree $\delta$ and discriminant $\Delta$. We will use the following bound on the product of the class number and the regulator, proven by Lenstra [1992, Theorem 6.5].

Lemma 28. Suppose that $k \neq \mathbb{Q}$. Let $r_{2}$ denote the number of complex places of $k$ and let $C=(2 / \pi)^{r_{2}} \sqrt{|\Delta|}$. We have

$$
h_{k} R_{k} \leq \frac{C(\log C)^{\delta-1-r_{2}}(\delta-1+\log C)^{r_{2}}}{(\delta-1) !} .
$$


Let $S$ be a finite set of places of $k$ containing the archimedean places. Recall that $Q_{S}$ is the minimum value of

$$
h\left(u_{1}\right) \cdots h\left(u_{s-1}\right),
$$

where $s=|S|$ and $u_{1}, \ldots, u_{s-1}$ are generators of $0_{k, S}^{*}$ modulo roots of unity. For the $S$-regulator and $Q_{S}$, Bugeaud and Gyôry [1996, Lemmas 1 and 3] gave the bounds

$$
\begin{aligned}
& R_{S} \leq h_{k} R_{k} \prod_{v \in S \backslash S_{\infty}} \log N(v), \\
& Q_{S} \leq \frac{((s-1) !)^{2}}{2^{s-2} \delta^{s-1}} R_{S} .
\end{aligned}
$$

More crudely, letting $s=|S|$ and $N=\max _{v \in S} N(v)$, we have the estimates

$$
\begin{aligned}
R_{k} \leq h_{k} R_{k} & \leq \frac{\sqrt{|\Delta|}\left(\frac{1}{2} \log |\Delta|\right)^{\delta-1-r_{2}}\left(\delta-1+\frac{1}{2} \log |\Delta|\right)^{r_{2}}}{(\delta-1) !} \\
& \leq \frac{\sqrt{|\Delta|}\left(\delta-1+\frac{1}{2} \log |\Delta|\right)^{\delta-1}}{(\delta-1) !} \leq \frac{\sqrt{|\Delta|} 2^{\delta-1} \delta^{\delta-1}\left(\log ^{*}|\Delta|\right)^{\delta-1}}{(\delta-1) !} \\
& \leq \delta^{\delta} \sqrt{|\Delta|}\left(\log ^{*}|\Delta|\right)^{\delta-1}
\end{aligned}
$$

and

$$
\begin{aligned}
& R_{S} \leq \delta^{\delta}\left(\log ^{*} N\right)^{s-\delta / 2} \sqrt{|\Delta|}\left(\log ^{*}|\Delta|\right)^{\delta-1}, \\
& Q_{S} \leq 2^{2-s} s^{2 s-4} \delta^{\delta-s+1}\left(\log ^{*} N\right)^{s-\delta / 2} \sqrt{|\Delta|}\left(\log ^{*}|\Delta|\right)^{\delta-1} .
\end{aligned}
$$

6.5. Points in projective space. We first recall an inequality of Silverman relating the height of a point in projective space and the discriminant of its field of definition. Theorem 29 [Silverman 1984, Theorem 2]. Let $k$ be a number field of degree $\delta$ and discriminant $\Delta$. Let $P \in \mathbb{P}^{n}(k)$. Then

$$
\frac{\log |\Delta|}{\delta} \leq(2 \delta-2) h(P)+\log \delta .
$$

For a number field $k$ and finite set of places $S$ of $k$ containing the archimedean places, define

$$
c_{3}(k, S)=\left\{\begin{array}{cl}
0 & \text { if } \delta=1 \text { or } s=1, \\
\frac{2 s ! s^{s+\frac{1}{2}} R_{S}}{\left(\log \delta / 6 \delta^{3}\right)^{s-2}} & \text { otherwise, }
\end{array}\right.
$$

where $s=|S|$. If $S_{\infty}$ denotes the set of archimedean places of $k$, then we let $c_{3}(k)=c_{3}\left(k, S_{\infty}\right)$.

The next lemma describes certain choices of coordinates for a point in projective space. 
Lemma 30. Let $k$ be a number field of degree $\delta, s$ the number of archimedean places of $k$, and $P \in \mathbb{P}^{n}(k)$.

(a) There exists a choice of homogeneous coordinates $P=\left(x_{0}, \ldots, x_{n}\right)$ such that $x_{0}, \ldots, x_{n} \in \mathcal{O}_{k}$ and for any $v \in M_{k}$,

$$
\begin{aligned}
\frac{1}{s} h(P)-c_{3}(k) & \leq \log \max \left\{\left\|x_{0}\right\|_{v}, \ldots,\left\|x_{n}\right\|_{v}\right\} \\
& \leq \frac{1}{s} h(P)+\frac{1}{2 \delta s} \log |\Delta|+c_{3}(k) \quad \text { if } v \mid \infty, \\
-\frac{1}{2 \delta} \log |\Delta| & \leq \log \max \left\{\left\|x_{0}\right\|_{v}, \ldots,\left\|x_{n}\right\|_{v}\right\} \leq 0 \quad \text { if } v \nmid \infty .
\end{aligned}
$$

(b) There exists a choice of homogeneous coordinates $P=\left(x_{0}, \ldots, x_{n}\right)$ such that $x_{0}, \ldots, x_{n} \in \mathbb{O}_{k}$ and for any $v \in M_{k}$,

$$
\begin{aligned}
0 & \leq \log \max \left\{\left\|x_{0}\right\|_{v}, \ldots,\left\|x_{n}\right\|_{v}\right\} & & \\
& \leq(2 \delta+1) h(P)+\log \delta & & \text { if } v \mid \infty, \\
-\delta^{2} h(P)-\frac{\delta}{2} \log \delta & \leq \log \max \left\{\left\|x_{0}\right\|_{v}, \ldots,\left\|x_{n}\right\|_{v}\right\} \leq 0 & & \text { if } v \nmid \infty .
\end{aligned}
$$

Proof. Let $S_{\infty}$ denote the set of archimedean places of $k$. The case $k=\mathbb{Q}$ follows immediately by choosing $x_{0}, \ldots, x_{n}$ to be integers with $\operatorname{gcd}\left(x_{0}, \ldots, x_{n}\right)=1$ and $P=\left(x_{0}, \ldots, x_{n}\right)$. We assume from now on that $\delta>1$. Let $P=\left(x_{0}, \ldots, x_{n}\right)$ be some choice of homogeneous coordinates with $x_{0}, \ldots, x_{n} \in \mathbb{O}_{k}$. Let $I$ be the ideal of $\mathbb{O}_{k}$ generated by $x_{0}, \ldots, x_{n}$. From the Minkowski bound, the ideal class of $I$ contains an (integral) ideal with norm $\leq \sqrt{|\Delta|}$. Thus, after rescaling $x_{0}, \ldots, x_{n}$, we may assume that the norm of $I$ satisfies $N(I) \leq \sqrt{|\Delta|}$. From the definition of the height, we have

$$
h(P)=\sum_{v \in M_{k}} \log \max \left\{\left\|x_{0}\right\|_{v}, \ldots,\left\|x_{n}\right\|_{v}\right\}=\sum_{v \in S_{\infty}} \log \max _{i}\left\|x_{i}\right\|_{v}-\frac{1}{\delta} \log N(I) .
$$

So

$$
h(P) \leq \sum_{v \in S_{\infty}} \log \max \left\{\left\|x_{0}\right\|_{v}, \ldots,\left\|x_{n}\right\|_{v}\right\} \leq h(P)+\frac{1}{2 \delta} \log |\Delta| .
$$

We first consider (a). The case $s=\left|S_{\infty}\right|=1$ is immediate from the above, so we assume from now on that $s>1$. Consider the image of the unit group $0_{k}^{*}$ via the logarithmic map $\lambda: 0_{k}^{*} \mapsto \mathbb{R}^{s}, \lambda(u)=\left(\log \|u\|_{v}\right)_{v \in S_{\infty}}$. The image is a lattice in the hyperplane of $\mathbb{R}^{s}$ defined by $\sum_{v \in S_{\infty}} x_{v}=0$. From [Hajdu 1993, p. 5], there exists a fundamental domain of this lattice with diameter $\leq 2 s ! s^{s+\frac{1}{2}} R_{k} /\left(\log \delta / 6 \delta^{3}\right)^{s-2}$. Let $c=\sum_{v \in S_{\infty}} \log \max \left\{\left\|x_{0}\right\|_{v}, \ldots,\left\|x_{n}\right\|_{v}\right\}$ and consider the vector

$$
\boldsymbol{v}=\left(\log \max \left\{\left\|x_{0}\right\|_{v}, \ldots,\left\|x_{n}\right\|_{v}\right\}-c / s\right)_{v \in S_{\infty}} .
$$


Then there exists a unit $u \in 0_{k}^{*}$ such that

$$
|\boldsymbol{v}-\lambda(u)| \leq \frac{2 s ! s^{s+\frac{1}{2}} R_{k}}{\left(\log \delta / 6 \delta^{3}\right)^{s-2}}=c_{3}(k) .
$$

Therefore, for every $v \in S_{\infty}$,

$$
\left|\log \max \left\{\left\|u^{-1} x_{0}\right\|_{v}, \ldots,\left\|u^{-1} x_{n}\right\|_{v}\right\}-c / s\right| \leq c_{3}(k)
$$

and

$\frac{1}{s} h(P)-c_{3}(k) \leq \log \max \left\{\left\|u^{-1} x_{0}\right\|_{v}, \ldots,\left\|u^{-1} x_{n}\right\|_{v}\right\} \leq \frac{1}{s} h(P)+\frac{1}{2 \delta s} \log |\Delta|+c_{3}(k)$.

Note that if $v \nmid \infty$, we also have

$$
-\frac{1}{2 \delta} \log |\Delta| \leq-\frac{1}{\delta} \log N(I) \leq \log \max \left\{\left\|x_{0}\right\|_{v}, \ldots,\left\|x_{n}\right\|_{v}\right\} \leq 0 .
$$

We now prove (b). From our earlier choice of coordinates, we have in particular

$$
\sum_{v \in S_{\infty}} \log \left\|x_{0}\right\|_{v}=\frac{1}{\delta} \log \left|N_{\mathbb{Q}}^{k}\left(x_{0}\right)\right| \leq h(P)+\frac{1}{2 \delta} \log |\Delta| .
$$

Then after scaling by $N_{\mathbb{Q}}^{k}\left(x_{0}\right) / x_{0} \in \mathscr{O}_{k}$, we may take $P=\left(x_{0}, \ldots, x_{n}\right)$ where $x_{0} \in \mathbb{Z}$,

$$
\frac{1}{\delta} \log \left|x_{0}\right| \leq h(P)+\frac{1}{2 \delta} \log |\Delta| \leq \delta h(P)+\frac{1}{2} \log \delta
$$

by Theorem 29, and $x_{1}, \ldots, x_{n} \in \mathcal{O}_{k}$. Let $v \in S_{\infty}$. Then $\log \max _{i}\left\|x_{i}\right\|_{v} \geq 0$ and

$$
\begin{aligned}
\log \max \left\{\left\|x_{0}\right\|_{v}, \ldots,\left\|x_{n}\right\|_{v}\right\} & =h(P)-\sum_{w \in M_{k} \backslash\{v\}} \log \max \left\{\left\|x_{0}\right\|_{w}, \ldots,\left\|x_{n}\right\|_{w}\right\} \\
& \leq h(P)-\sum_{w \in M_{k} \backslash\{v\}} \log \left\|x_{0}\right\|_{w} \leq h(P)+\log \left\|x_{0}\right\|_{v} \\
& \leq h(P)+2\left(\delta h(P)+\frac{1}{2} \log \delta\right) \leq(2 \delta+1) h(P)+\log \delta .
\end{aligned}
$$

We also clearly have

$$
-\delta^{2} h(P)-\frac{\delta}{2} \log \delta \leq-\log \left|x_{0}\right| \leq \log \max \left\{\left\|x_{0}\right\|_{v}, \ldots,\left\|x_{n}\right\|_{v}\right\} \leq 0
$$

if $v$ is nonarchimedean.

We also need the following result from the main theorem of [Hajdu 1993], which is closely related to the previous lemma.

Theorem 31. Let $k$ be a number field of degree $\delta$ and let $S$ be a finite set of places of $k$ containing the archimedean places. Let $\alpha \in k$. Then we can write

$$
\alpha=\beta u,
$$


where $u \in \mathrm{O}_{k, S}^{*}$ and

$$
h(\beta)<s c_{3}(k, S)+\sum_{v \notin S} h_{v}(\alpha)+\sum_{v \in S} \log \|\alpha\|_{v}<s c_{3}(k, S)+\sum_{v \notin S} h_{v}(\alpha)+h_{v}(1 / \alpha) .
$$

The last inequality follows from the product formula. This result is actually only proven in [ibid.] for $S$-integers $\alpha$, but the same proof given there yields the result above.

We note the estimates

$$
\begin{aligned}
c_{3}(k, S) & \leq 2^{4 s} s^{2 s} \delta^{3 s+\delta-6} \sqrt{|\Delta|}\left(\log ^{*}|\Delta|\right)^{\delta-1}\left(\log ^{*} N\right)^{s-\delta / 2}, \\
c_{3}(k) & \leq 2^{4 \delta} \delta^{6 \delta-6 \sqrt{|\Delta|}}\left(\log ^{*}|\Delta|\right)^{\delta-1} .
\end{aligned}
$$

6.6. Miscellaneous elementary estimates. We have the following lower bound for heights on $\mathbb{P}^{1}$.

Lemma 32. Let $S$ be a set of places of a number field $k$. Let $P, Q \in \mathbb{P}^{1}(k), P \neq Q$. Then

$$
\sum_{v \in S} h_{Q, v}(P) \geq-\log 2 .
$$

Proof. Let $P=\left(x_{1}, y_{1}\right), Q=\left(x_{2}, y_{2}\right), x_{1}, x_{2}, y_{1}, y_{2} \in k$. Then

$$
\begin{aligned}
h_{Q, v}(P) & =\log \frac{\max \left\{\left\|x_{1}\right\|_{v},\left\|y_{1}\right\|_{v}\right\} \max \left\{\left\|x_{2}\right\|_{v},\left\|y_{2}\right\|_{v}\right\}}{\left\|x_{1} y_{2}-x_{2} y_{1}\right\|_{v}} \\
& \geq \log \frac{\max \left\{\left\|x_{1}\right\|_{v},\left\|y_{1}\right\|_{v}\right\} \max \left\{\left\|x_{2}\right\|_{v},\left\|y_{2}\right\|_{v}\right\}}{\epsilon_{v}^{\prime}(2) \max \left\{\left\|x_{1} y_{2}\right\|_{v},\left\|x_{2} y_{1}\right\|_{v}\right.} \\
& \geq \log \frac{\max \left\{\left\|x_{1}\right\|_{v},\left\|y_{1}\right\|_{v}\right\} \max \left\{\left\|x_{2}\right\|_{v},\left\|y_{2}\right\|_{v}\right\}}{\epsilon_{v}^{\prime}(2) \max \left\{\left\|x_{1}\right\|_{v},\left\|y_{1}\right\|_{v}\right\} \max \left\{\left\|x_{2}\right\|_{v},\left\|y_{2}\right\|_{v}\right\}} \\
& \geq-\log \epsilon_{v}^{\prime}(2) .
\end{aligned}
$$

Therefore, $\sum_{v \in S} h_{Q, v}(P) \geq-\log 2$.

We need an estimate on the height of a product of polynomials [Hindry and Silverman 2000, Proposition B.7.4].

Lemma 33. Let $k$ be a number field. Let $f_{1}, \ldots, f_{m} \in k\left[x_{1}, \ldots, x_{n}\right]$ be polynomials and let $f=f_{1} \cdots f_{m}$. Then for any $v \in M_{k}$,

$$
|f|_{v} \leq \epsilon_{v}\left(\prod_{i=2}^{m} 2^{\operatorname{deg} f_{i}}\right) \prod_{i=1}^{m}\left|f_{i}\right|_{v}
$$

In particular,

$$
h(f) \leq \sum_{i=1}^{m} h\left(f_{i}\right)+\left(\sum_{i=2}^{m} \operatorname{deg} f_{i}\right) \log 2
$$


For maps between projective spaces, we have the following height inequality [Hindry and Silverman 2000, p. 181].

Lemma 34. Let $\phi: \mathbb{P}^{n} \rightarrow \mathbb{P}^{m}$ be a rational map of degree d defined over $\overline{\mathbb{Q}}$. Then

$$
h(\phi(P)) \leq d h(P)+h(\phi)+\log \left(\begin{array}{c}
n+d \\
n
\end{array}\right)
$$

for all $P \in \mathbb{P}^{n}(\overline{\mathbb{Q}})$ where $\phi$ is defined.

We also need an elementary estimate for polynomials in two variables.

Lemma 35. Let $k$ be a number field. Let $f \in k[x, y]$ be a polynomial of degree $d$ and let $v \in M_{k}$. Let $a, b, x, y \in k$ and suppose that $|x-a|_{v},|y-b|_{v} \leq 1$. Then $|f(x, y)-f(a, b)|_{v} \leq \epsilon_{v}\left((d+2)^{4} 2^{d}\right)|f|_{v} \max \left\{|a|_{v},|b|_{v}, 1\right\}^{d} \max \left\{|x-a|_{v},|y-b|_{v}\right\}$. Proof. Let $f(x, y)=\sum c_{i j} x^{i} y^{j}$. Looking at the Taylor series for $f(x, y)$ around $(a, b)$ and applying the triangle inequality, we find

$$
\begin{aligned}
\mid f(x, y)- & \left.f(a, b)\right|_{v} \leq\left|\sum_{m, n, m+n>0}\left(\frac{\partial^{m+n} f}{\partial x^{m} \partial y^{n}}\right)(a, b) \frac{(x-a)^{m}(y-b)^{n}}{m ! n !}\right|_{v} \\
& \leq \epsilon_{v}\left(\left(\begin{array}{c}
d+2 \\
2
\end{array}\right)\right) \max _{m, n}\left|\frac{1}{m ! n !}\left(\frac{\partial^{m+n} f}{\partial x^{m} \partial y^{n}}\right)(a, b)\right|_{v} \max \left\{|x-a|_{v},|y-b|_{v}\right\} .
\end{aligned}
$$

Since

$$
\begin{aligned}
\left|\frac{1}{m ! n !}\left(\frac{\partial^{m+n} f}{\partial x^{m} \partial y^{n}}\right)(a, b)\right|_{v} & =\left|\sum c_{i j}\left(\begin{array}{c}
i \\
m
\end{array}\right)\left(\begin{array}{c}
j \\
n
\end{array}\right) a^{i-m} b^{j-n}\right|_{v} \\
& \leq \epsilon_{v}\left(\left(\begin{array}{c}
d+2 \\
2
\end{array}\right)\right) \max _{i, j}\left|c_{i j}\right|_{v}\left|\left(\begin{array}{c}
i \\
m
\end{array}\right)\left(\begin{array}{c}
j \\
n
\end{array}\right)\right|_{v}\left|a^{i-m} b^{j-n}\right|_{v} \\
& \leq \epsilon_{v}\left(\left(\begin{array}{c}
d+2 \\
2
\end{array}\right) 2^{d}\right)|f|_{v} \max \left\{|a|_{v},|b|_{v}, 1\right\}^{d},
\end{aligned}
$$

we have

$|f(x, y)-f(a, b)|_{v}$

$$
\leq \epsilon_{v}\left((d+2)^{4} 2^{d}\right)|f|_{v} \max \left\{|a|_{v},|b|_{v}, 1\right\}^{d} \max \left\{|x-a|_{v},|y-b|_{v}\right\} .
$$

Finally, we prove an explicit version of Lemma 10 when $X=\mathbb{P}^{2}$.

Lemma 36. Let $k$ be a number field and let $\phi \in k\left(\mathbb{P}^{2}\right)$ be a rational function of degree $d$ on $\mathbb{P}^{2}$. Let $P, Q \in \mathbb{P}^{2}(k) \backslash \operatorname{Supp} \phi$ and $T \subset M_{k}$. Suppose that $\phi(P) \neq \phi(Q)$. Then

$\sum_{v \in T} h_{Q, v}(P) \leq \sum_{v \in T} h_{\phi(Q), v}(\phi(P))+(2 d+2) h(Q)+8 \log (d+2)+(2 d+4) \log 2$. 
Proof. Let $\phi=f_{1} / f_{2}$, where $f_{1}, f_{2} \in \mathcal{O}_{k}[x, y, z]$ are homogeneous polynomials of degree $d$. Let $Q=\left(x_{0}, y_{0}, z_{0}\right), P=(x, y, z)$, and $\alpha=\phi(Q)$. From the definitions,

$$
\begin{aligned}
h_{Q, v}(P) & =\log \frac{\max \left\{\left\|x_{0}\right\|_{v},\left\|y_{0}\right\|_{v},\left\|z_{0}\right\|_{v}\right\} \max \left\{\|x\|_{v},\|y\|_{v},\|z\|_{v}\right\}}{\max \left\{\left\|z_{0} x-x_{0} z\right\|_{v},\left\|z_{0} y-y_{0} z\right\|_{v},\left\|x_{0} y-y_{0} x\right\|_{v}\right\}}, \\
h_{\alpha, v}(\phi(P)) & =\log \frac{\max \left\{\|\alpha\|_{v}, 1\right\} \max \left\{\left\|f_{1}(x, y, z)\right\|_{v},\left\|f_{2}(x, y, z)\right\|_{v}\right\}}{\left\|f_{1}(x, y, z)-\alpha f_{2}(x, y, z)\right\|_{v}} .
\end{aligned}
$$

Without loss of generality, after permuting the variables, we can assume that $z_{0} \neq 0$ and $Q=\left(x_{0}, y_{0}, 1\right)$. If $z=0$, then

$$
\begin{aligned}
h_{Q, v}(P) & =\log \frac{\max \left\{\left\|x_{0}\right\|_{v},\left\|y_{0}\right\|_{v}, 1\right\} \max \left\{\|x\|_{v},\|y\|_{v}\right\}}{\max \left\{\|x\|_{v},\|y\|_{v},\left\|x_{0} y-y_{0} x\right\|_{v}\right\}} \\
& \leq \log \max \left\{\left\|x_{0}\right\|_{v},\left\|y_{0}\right\|_{v}, 1\right\} .
\end{aligned}
$$

So

$$
\sum_{v \in T} h_{Q, v}(P) \leq \sum_{v \in T} \log \max \left\{\left\|x_{0}\right\|_{v},\left\|y_{0}\right\|_{v}, 1\right\} \leq h(Q) .
$$

Then using Lemma 32, in this case we have

$$
\sum_{v \in T} h_{Q, v}(P) \leq \sum_{v \in T} h_{\alpha, v}(P)+h(Q)+\log 2 .
$$

Suppose now that $z \neq 0$, in which case we can take $P=(x, y, 1)$, for some $x, y \in k$.

First suppose that

$$
\max \left\{\left|x-x_{0}\right|_{v},\left|y-y_{0}\right|_{v}\right\}<\frac{1}{\epsilon_{v}\left((d+2)^{4} 2^{d+1}\right)} \min _{j=1,2} \frac{\left|f_{j}\left(x_{0}, y_{0}, 1\right)\right|_{v}}{\left|f_{j}\right|_{v} \max \left\{\left|x_{0}\right|_{v},\left|y_{0}\right|_{v}, 1\right\}^{d}} .
$$

In particular, $\max \left\{\left|x-x_{0}\right|_{v},\left|y-y_{0}\right|_{v}\right\} \leq 1$. Let $F(u, v)=f_{1}(u, v, 1)-\alpha f_{2}(u, v, 1)$. Note that $\operatorname{deg} F \leq d$. From the definition of $\alpha, F\left(x_{0}, y_{0}\right)=0$. Then by Lemma 35, with $a=x_{0}, b=y_{0}$, we have

$$
\begin{aligned}
& |F(x, y)|_{v} \\
& \quad \leq \epsilon_{v}\left((d+2)^{4} 2^{d}\right)|F|_{v} \max \left\{\left|x_{0}\right|_{v},\left|y_{0}\right|_{v}, 1\right\}^{d} \max \left\{\left|x-x_{0}\right|_{v},\left|y-y_{0}\right|_{v}\right\} .
\end{aligned}
$$

For $j=1,2$, using Lemma 35 again, we find, if $v$ is archimedean,

$\left|f_{j}(x, y, 1)\right|_{v}$

$\geq\left|f_{j}\left(x_{0}, y_{0}, 1\right)\right|_{v}-(d+2)^{4} 2^{d}\left|f_{j}\right|_{v} \max \left\{\left|x_{0}\right|_{v},\left|y_{0}\right|_{v}, 1\right\}^{d} \max \left\{\left|x-x_{0}\right|_{v},\left|y-y_{0}\right|_{v}\right\}$

$\geq \frac{1}{2}\left|f_{j}\left(x_{0}, y_{0}, 1\right)\right|_{v}$.

By the same reasoning, if $v$ is nonarchimedean we have

$$
\left|f_{j}(x, y, 1)-f_{j}\left(x_{0}, y_{0}, 1\right)\right|_{v}<\left|f_{j}\left(x_{0}, y_{0}, 1\right)\right|_{v},
$$


and so

$$
\left|f_{j}(x, y, 1)\right|_{v}=\left|f_{j}\left(x_{0}, y_{0}, 1\right)\right|_{v} \quad \text { for } j=1,2 .
$$

Then in any case,

$$
\left|f_{j}(x, y, 1)\right|_{v} \geq \frac{1}{\epsilon_{v}(2)}\left|f_{j}\left(x_{0}, y_{0}, 1\right)\right|_{v} \quad \text { for } j=1,2 .
$$

Since $\max \left\{\left|x-x_{0}\right|_{v},\left|y-y_{0}\right|_{v}\right\} \leq 1$, we also have

$$
\max \left\{|x|_{v},|y|_{v}, 1\right\} \leq \epsilon_{v}(2) \max \left\{\left|x_{0}\right|_{v},\left|y_{0}\right|_{v}, 1\right\} .
$$

Then

$$
\begin{aligned}
h_{Q, v}(P) & =\log \frac{\max \left\{\left\|x_{0}\right\|_{v},\left\|y_{0}\right\|_{v}, 1\right\} \max \left\{\|x\|_{v},\|y\|_{v}, 1\right\}}{\max \left\{\left\|x-x_{0}\right\|_{v},\left\|y-y_{0}\right\|_{v},\left\|x_{0} y-y_{0} x\right\|_{v}\right\}} \\
& \leq 2 \log \max \left\{\left\|x_{0}\right\|_{v},\left\|y_{0}\right\|_{v}, 1\right\}+\log \epsilon_{v}^{\prime}(2)-\log \max \left\{\left\|x-x_{0}\right\|_{v},\left\|y-y_{0}\right\|_{v}\right\}
\end{aligned}
$$

and

$$
\begin{aligned}
h_{\alpha, v}(\phi(P)) & =\log \frac{\max \left\{\|\alpha\|_{v}, 1\right\} \max \left\{\left\|f_{1}(x, y, 1)\right\|_{v},\left\|f_{2}(x, y, 1)\right\|_{v}\right\}}{\left\|f_{1}(x, y, 1)-\alpha f_{2}(x, y, 1)\right\|_{v}} \\
& =\log \max _{j=1,2}\left\|f_{j}(x, y, 1)\right\|_{v}+\log \max \left\{\|\alpha\|_{v}, 1\right\}-\log \|F(x, y)\|_{v} \\
& \geq \log \max _{j=1,2}\left\|f_{j}\left(x_{0}, y_{0}, 1\right)\right\|_{v}+\log \max \left\{\|\alpha\|_{v}, 1\right\}-\epsilon_{v}^{\prime}\left(\log (d+2)^{4} 2^{d+1}\right) \\
& -\log \|F\|_{v}-d \log \max \left\{\left\|x_{0}\right\|_{v},\left\|y_{0}\right\|_{v}, 1\right\}-\log \max \left\{\left\|x-x_{0}\right\|_{v},\left\|y-y_{0}\right\|_{v}\right\}
\end{aligned}
$$

by (6). We can write $|F|_{v}=\left|f_{1}-\alpha f_{2}\right|_{v} \leq \epsilon_{v}(2) \max \left\{\left|f_{1}\right|_{v},\left|f_{2}\right|_{v}\right\} \max \left\{|\alpha|_{v}, 1\right\}$. So $h_{\alpha, v}(\phi(P)) \geq \log \max _{j=1,2}\left\|f_{j}\left(x_{0}, y_{0}, 1\right)\right\|_{v}-\epsilon_{v}^{\prime}\left(\log (d+2)^{4} 2^{d+2}\right)-\log \max _{j=1,2}\left\|f_{j}\right\|_{v}$ $-d \log \max \left\{\left\|x_{0}\right\|_{v},\left\|y_{0}\right\|_{v}, 1\right\}-\log \max \left\{\left\|x-x_{0}\right\|_{v},\left\|y-y_{0}\right\|_{v}\right\}$.

Note that

$$
\left|f_{j}\left(x_{0}, y_{0}, 1\right)\right|_{v} \leq \epsilon_{v}\left(\left(\begin{array}{c}
d+2 \\
2
\end{array}\right)\right)\left|f_{j}\right|_{v} \max \left\{\left|x_{0}\right|_{v},\left|y_{0}\right|_{v}, 1\right\}^{d} \quad \text { for } j=1,2 .
$$

This implies that

$$
\begin{gathered}
\sum_{v \in T} \log \frac{\max \left\{\left\|f_{1}\left(x_{0}, y_{0}, 1\right)\right\|_{v},\left\|f_{2}\left(x_{0}, y_{0}, 1\right)\right\|_{v}\right\}}{\max \left\{\left\|f_{1}\right\|_{v},\left\|f_{2}\right\|_{v}\right\} \max \left\{\left\|x_{0}\right\|_{v},\left\|y_{0}\right\|_{v}, 1\right\}^{d}} \\
\geq \sum_{j=1}^{2} \sum_{v \in M_{k}} \log \frac{\left\|f_{j}\left(x_{0}, y_{0}, 1\right)\right\|_{v}}{\left\|f_{j}\right\|_{v} \max \left\{\left\|x_{0}\right\|_{v},\left\|y_{0}\right\|_{v}, 1\right\}^{d}}-2 \log \epsilon_{v}^{\prime}\left(\left(\begin{array}{c}
d+2 \\
2
\end{array}\right)\right) \\
\geq-2 d h(Q)-4 \log (d+2)
\end{gathered}
$$


by the product formula. So

$$
\begin{aligned}
& \sum_{v \in T} h_{\alpha, v}(\phi(P)) \\
& \geq-2 d h(Q)-8 \log (d+2)-(d+2) \log 2-\sum_{v \in T} \log \max \left\{\left\|x-x_{0}\right\|_{v},\left\|y-y_{0}\right\|_{v}\right\} .
\end{aligned}
$$

Then

$$
\sum_{v \in T} h_{Q, v}(P) \leq \sum_{v \in T} h_{\alpha, v}(\phi(P))+(2 d+2) h(Q)+8 \log (d+2)+(d+3) \log 2 .
$$

Finally, suppose that

$$
\max \left\{\left|x-x_{0}\right|_{v},\left|y-y_{0}\right|_{v}\right\} \geq C_{v}
$$

where

$$
C_{v}=\frac{1}{\epsilon_{v}\left((d+2)^{4} 2^{d+1}\right)} \min \left\{\frac{\left|f_{1}\left(x_{0}, y_{0}, 1\right)\right|_{v}}{\left|f_{1}\right|_{v} \max \left\{\left|x_{0}\right|_{v},\left|y_{0}\right|_{v}, 1\right\}^{d}}, \frac{\left|f_{2}\left(x_{0}, y_{0}, 1\right)\right|_{v}}{\left|f_{2}\right|_{v} \max \left\{\left|x_{0}\right|_{v},\left|y_{0}\right|_{v}, 1\right\}^{d}}\right\} .
$$

As noted before, $C_{v} \leq 1$. Then one easily finds that

$$
\begin{aligned}
\frac{\max \left\{|x|_{v},|y|_{v}, 1\right\}}{\max \left\{\left|x-x_{0}\right|_{v},\left|y-y_{0}\right|_{v}\right\}} & =\frac{\max \left\{\left|\left(x-x_{0}\right)+x_{0}\right|_{v},\left|\left(y-y_{0}\right)+y_{0}\right|_{v}, 1\right\}}{\max \left\{\left|x-x_{0}\right|_{v},\left|y-y_{0}\right|_{v}\right\}} \\
& \leq \frac{\epsilon_{v}(2) \max \left\{\left|\left(x-x_{0}\right)\right|_{v},\left|\left(y-y_{0}\right)\right|_{v},\left|x_{0}\right|_{v},\left|y_{0}\right|_{v}, 1\right\}}{\max \left\{\left|x-x_{0}\right|_{v},\left|y-y_{0}\right|_{v}\right\}} \\
& \leq \frac{\epsilon_{v}(2) \max \left\{\left|x_{0}\right|_{v},\left|y_{0}\right|_{v}, 1\right\}}{C_{v}}
\end{aligned}
$$

So

$$
\begin{aligned}
h_{Q, v}(P) & =\frac{\left[k_{v}: \mathbb{Q}_{v}\right]}{[k: \mathbb{Q}]} \log \frac{\max \left\{\left|x_{0}\right|_{v},\left|y_{0}\right|_{v}, 1\right\} \max \left\{|x|_{v},|y|_{v}, 1\right\}}{\max \left\{\left|x-x_{0}\right|_{v},\left|y-y_{0}\right|_{v},\left|x_{0} y-y_{0} x\right|_{v}\right\}} \\
& \leq \frac{\left[k_{v}: \mathbb{Q}_{v}\right]}{[k: \mathbb{Q}]} \log \epsilon_{v}(2) \max \left\{\left|x_{0}\right|_{v},\left|y_{0}\right|_{v}, 1\right\}^{2} / C_{v} \\
& \leq \frac{\left[k_{v}: \mathbb{Q}_{v}\right]}{[k: \mathbb{Q}]}\left(2 \log \max \left\{\left|x_{0}\right|_{v},\left|y_{0}\right|_{v}, 1\right\}+\log \epsilon_{v}(2)-\log C_{v}\right) .
\end{aligned}
$$

Then using Lemma 32, we find

$$
\sum_{v \in T} h_{Q, v}(P) \leq \sum_{v \in T} h_{\alpha, v}(\phi(P))+2 h(Q)+2 \log 2-\sum_{v \in T} \frac{\left[k_{v}: \mathbb{Q}_{v}\right]}{[k: \mathbb{Q}]} \log C_{v}
$$


Since

$$
\begin{aligned}
\sum_{v \in T} \frac{\left[k_{v}: \mathbb{Q}_{v}\right]}{[k: \mathbb{Q}]} \log C_{v} & \geq \sum_{v \in M_{k}} \frac{\left[k_{v}: \mathbb{Q}_{v}\right]}{[k: \mathbb{Q}]} \log C_{v} \\
& \geq \sum_{j=1}^{2} \sum_{v \in M_{k}} \log \frac{\left\|f_{j}\left(x_{0}, y_{0}, 1\right)\right\|_{v}}{\epsilon_{v}^{\prime}\left((d+2)^{4} 2^{d+1}\right)\left\|f_{j}\right\|_{v} \max \left\{\left\|x_{0}\right\|_{v},\left\|y_{0}\right\|_{v}, 1\right\}^{d}} \\
& \geq-8 \log (d+2)-(2 d+2) \log 2-2 d h(Q),
\end{aligned}
$$

where we have used the product formula in the last line, we obtain

$$
\sum_{v \in T} h_{Q, v}(P) \leq \sum_{v \in T} h_{\alpha, v}(\phi(P))+(2 d+2) h(Q)+(2 d+4) \log 2+8 \log (d+2) .
$$

\section{Explicit results for $\mathbb{P}^{2}$}

In this section we give a proof of Theorem 7. The proof will follow the proof in Section 3.3, except that we will give explicit estimates at each step. We begin with an explicit version of Theorem 19.

Theorem 37. Let $k$ be a number field of degree $\delta$ and discriminant $\Delta$. Let $S$ be a finite set of places of $k$, containing the archimedean places, of cardinality $s$. Let $C_{1}$ and $C_{2}$ be distinct curves over $k$ in $\mathbb{P}^{2}$ defined by homogeneous polynomials $f_{1}, f_{2} \in \mathbb{O}_{k}[x, y, z]$, respectively, of degrees $d_{1}$ and $d_{2}$, respectively. Let $d=$ $\max \left\{d_{1}, d_{2}\right\}$ and $\phi=f_{1}^{d_{2}}(x, y, z) / f_{2}^{d_{1}}(x, y, z)$, a rational function on $\mathbb{P}^{2}$. Let $Q \in \mathbb{P}^{2}(\bar{k}) \backslash\left(C_{1} \cup C_{2}\right)$ and let $\delta^{\prime}=[k(Q): \mathbb{Q}]$. Let $w \in M_{k(Q)}$ and let $0<\epsilon<1$. Then for all $P \in\left(\mathbb{P}^{2} \backslash\left(C_{1} \cup C_{2}\right)\right)\left(\mathbb{O}_{k, S}\right)$, either

$$
h_{Q, w}(P) \leq \epsilon h(P)+c_{4}\left(\epsilon, k, S, w, Q, C_{1}, C_{2}\right)
$$

or

$$
\phi(P)=\phi(Q),
$$

where

$$
\begin{aligned}
& c_{4}=\left(2 d^{2}+2\right) h(Q)+10 \log \left(d^{2}+2\right)+\left(2 d^{2}+7\right) \log 2 \\
& +\frac{1}{d}\left(h\left(C_{1}\right)+h\left(C_{2}\right)\right)+\frac{\log |\Delta|}{\delta d^{2}}+\frac{2 \delta}{d^{2}} c_{3}(k)+c_{5}, \\
& c_{5}=6.4\left(d^{2} c_{2}\left(\delta^{\prime}, s\right) / \epsilon\right) \frac{N(w)}{\log N(w)} c_{6} c_{7} \max \left\{\log \left(\left(d^{2} c_{2}\left(\delta^{\prime}, s\right) / \epsilon\right) N(w)\right), \log ^{*} c_{6}\right\}, \\
& c_{6}=Q_{S}\left(1+s c_{3}(k, S)+\frac{1}{\delta} \log |\Delta|\right), \\
& c_{7}=d^{2} h(Q)+d h\left(C_{1}\right)+d h\left(C_{2}\right)+\frac{1}{\delta} \log |\Delta|+2 \delta c_{3}(k)+2 d^{2} \log 2+2 \log \left(d^{2}+2\right) \text {. }
\end{aligned}
$$


In particular, (7) holds for all $P \in\left(\mathbb{P}^{2} \backslash\left(C_{1} \cup C_{2}\right)\right)\left(\mathbb{O}_{k, S}\right)$ outside of an effectively computable finite union of plane curves $Z$.

Proof. Let $I_{1}$ and $I_{2}$ be the ideals of $\mathrm{O}_{k}$ generated by the coefficients of $g_{1}=f_{1}^{d_{2}}$ and $g_{2}=f_{2}^{d_{1}}$, respectively. We rescale $g_{1}$ and $g_{2}$ as in Lemma 30(a) and its proof (viewing the coefficients of the polynomials as giving points in projective space). In particular, $N\left(I_{1}\right), N\left(I_{2}\right) \leq \sqrt{|\Delta|}$. Let $\phi=g_{1} / g_{2}$ and let $P \in\left(\mathbb{P}^{2} \backslash\left(C_{1} \cup C_{2}\right)\right)\left(\mathbb{O}_{k, S}\right)$. Then it follows from the definitions that we have an equality of fractional ideals $\phi(P) O_{k}=\left(I_{1} / I_{2}\right) J$, where $J$ is a fractional ideal supported on the primes in $S$. By Theorem 31, we can write $\phi(P)=\beta u$, where $u \in O_{k, S}^{*}$ and

$$
h(\beta) \leq s c_{3}(k, S)+\frac{1}{\delta} \log N\left(I_{1}\right)+\frac{1}{\delta} \log N\left(I_{2}\right) \leq s c_{3}(k, S)+\frac{1}{\delta} \log |\Delta| .
$$

Let $\alpha=\phi(Q)$ and suppose that $\phi(P) \neq \alpha$. By Theorem 24, substituting $\epsilon / d^{2}$ for $\epsilon$ and taking $G$ to be the multiplicative group generated by $\beta$ and $O_{k, S}^{*}$, we have the inequality

$$
h_{\alpha, w}(\phi(P)) \leq \frac{\epsilon}{d^{2}} h(\phi(P))+c_{1}\left(\frac{\epsilon}{d^{2}}, k(Q), G, w, \alpha\right)+\log 2 .
$$

Note that $\operatorname{deg} \phi \leq d^{2}$. By Lemma 36,

$$
\begin{aligned}
& h_{Q, w}(P) \leq h_{\alpha, w}(\phi(P))+\left(2 d^{2}+2\right) h(Q)+8 \log \left(d^{2}+2\right)+\left(2 d^{2}+4\right) \log 2 \\
& \leq \frac{\epsilon}{d^{2}} h(\phi(P))+\left(2 d^{2}+2\right) h(Q)+8 \log \left(d^{2}+2\right) \\
& \\
&+\left(2 d^{2}+5\right) \log 2+c_{1}\left(\frac{\epsilon}{d^{2}}, k(Q), G, w, \alpha\right) .
\end{aligned}
$$

By Lemma 34,

$$
h(\phi(P)) \leq d^{2} h(P)+h(\phi)+\log \left(\begin{array}{c}
d^{2}+2 \\
2
\end{array}\right) \leq d^{2} h(P)+h(\phi)+2 \log \left(d^{2}+2\right) .
$$

Let $s_{\infty}$ be the number of archimedean places of $k$. By Lemma 30(a) and the construction of $g_{1}$ and $g_{2}$,

$$
\begin{aligned}
h(\phi) & =\sum_{v \in M_{k}} \log \max \left\{\left\|g_{1}\right\|_{v},\left\|g_{2}\right\|_{v}\right\} \leq \sum_{\substack{v \in M_{k} \\
v \mid \infty}} \log \max \left\{\left\|g_{1}\right\|_{v},\left\|g_{2}\right\|_{v}\right\} \\
& \leq s_{\infty}\left(\frac{1}{s_{\infty}} h\left(f_{1}^{d_{2}}\right)+\frac{1}{2 \delta s_{\infty}} \log |\Delta|+c_{3}(k)+\frac{1}{s_{\infty}} h\left(f_{2}^{d_{1}}\right)+\frac{1}{2 \delta s_{\infty}} \log |\Delta|+c_{3}(k)\right) \\
& \leq h\left(f_{1}^{d_{2}}\right)+h\left(f_{2}^{d_{1}}\right)+\frac{1}{\delta} \log |\Delta|+2 \delta c_{3}(k) .
\end{aligned}
$$

By Lemma 33,

$$
h\left(f_{1}^{d_{2}}\right)+h\left(f_{2}^{d_{1}}\right) \leq d h\left(f_{1}\right)+d h\left(f_{2}\right)+2 d^{2} \log 2=d h\left(C_{1}\right)+d h\left(C_{2}\right)+2 d^{2} \log 2 .
$$


So

$$
h(\phi) \leq d h\left(C_{1}\right)+d h\left(C_{2}\right)+\frac{1}{\delta} \log |\Delta|+2 \delta c_{3}(k)+2 d^{2} \log 2 .
$$

Then

$$
\begin{aligned}
& h_{Q, w}(P) \\
& <\epsilon h(P)+\left(2 d^{2}+2\right) h(Q)+10 \log \left(d^{2}+2\right)+\left(2 d^{2}+7\right) \log 2 \\
& \quad+\frac{1}{d}\left(h\left(C_{1}\right)+h\left(C_{2}\right)\right)+\frac{1}{\delta d^{2}} \log |\Delta|+\frac{2 \delta}{d^{2}} c_{3}(k)+c_{1}\left(\frac{\epsilon}{d^{2}}, k(Q), G, w, \alpha\right) .
\end{aligned}
$$

Finally, we can estimate the last term using

$$
Q_{G} \leq Q_{S} \max \{h(\beta), 1\} \leq Q_{S}\left(1+s c_{3}(k, S)+\frac{1}{\delta} \log |\Delta|\right)
$$

and, using Lemma 34 again,

$$
\begin{aligned}
h(\alpha) \leq d^{2} h(Q)+h(\phi)+2 \log \left(d^{2}+2\right) & \\
\leq d^{2} h(Q)+d h\left(C_{1}\right)+d h\left(C_{2}\right)+ & \frac{1}{\delta} \log |\Delta| \\
& +2 \delta c_{3}(k)+2 d^{2} \log 2+2 \log \left(d^{2}+2\right) .
\end{aligned}
$$

Proof of Theorem 7. Let $d_{i}=\operatorname{deg} C_{i}, i=1, \ldots, n$. Let $P \in\left(\mathbb{P}^{2} \backslash \bigcup_{i=1}^{n} C_{i}\right)\left(\mathbb{O}_{k, S}\right)$. Then

$$
\sum_{v \in S} h_{C_{i}, v}(P)=d_{i} h(P) \quad \text { for } i=1, \ldots, n .
$$

So for each $i$, there exists a place $v \in S$ such that $h_{C_{i}, v}(P) \geq(1 / s) h(P)$. Since $s<n$, there exists a place $v \in S$ and distinct elements $i, j \in\{1, \ldots, n\}$ such that

$$
\min \left\{h_{C_{i}, v}(P), h_{C_{j}, v}(P)\right\} \geq \frac{1}{s} h(P) .
$$

The theorem is then a consequence of the following lemma.

Lemma 38. Let $k$ be a number field of degree $\delta$ and discriminant $\Delta$. Let $S$ be a finite set of places of $k$, containing the archimedean places, of cardinality $s$. Let $C_{1}, \ldots, C_{n} \subset \mathbb{P}^{2}$ be distinct curves over $k$ such that at most $n-2$ of the curves $C_{i}$ intersect at any point of $\mathbb{P}^{2}(\bar{k})$. Let $d_{i}=\operatorname{deg} C_{i}, d=\max _{i} d_{i}, h=\max _{i} h\left(C_{i}\right)$, and $N=\max _{v \in S} N(v)$. Let $Z^{\prime}$ be the set from Theorem 7. Let $0<\epsilon<1$ and $v \in S$. Then any point $P \in\left(\mathbb{P}^{2} \backslash \bigcup_{i=1}^{n} C_{i}\right)\left(\mathscr{O}_{k, S}\right)$ with

$$
\min \left\{h_{C_{1}, v}(P), h_{C_{2}, v}(P)\right\} \geq \epsilon h(P)
$$

satisfies either $P \in Z^{\prime}$ or $h(P)<2^{20 s+4 \delta+75} d^{6 s+34} \delta^{5 s+8 \delta-3} s^{4 s-1} N^{d^{2}}\left(\log ^{*} N\right)^{2 s}|\Delta|^{3 / 2}\left(\log ^{*}|\Delta|\right)^{3 \delta}(h+1) / \epsilon^{3}$. 
Proof. Let

$$
\left(C_{1} \cap C_{2}\right)(\bar{k})=\left\{Q_{1}, \ldots, Q_{r}\right\} \subset \mathbb{P}^{2}(\bar{k})
$$

and let $Q_{i}=\left(x_{i}, y_{i}, z_{i}\right), x_{i}, y_{i}, z_{i} \in \mathcal{O}_{k\left(Q_{i}\right)}, i=1, \ldots, r$, where $r \leq d^{2}$. Let $L=k\left(Q_{1}, \ldots, Q_{r}\right)$. We note that $\left[k\left(Q_{i}\right): k\right] \leq d^{2}$ for all $i$. Let $C_{i}$ be defined by $f_{i} \in \mathfrak{O}_{k}[x, y, z], i=1, \ldots, n$, and let

$$
h_{\infty}=\log \max _{\substack{w \in M_{L} \\ w \mid \infty}}\left\{\left|f_{1}\right|_{w},\left|f_{2}\right|_{w}, \max \left|\prod_{i=1}^{r} g_{i}\right|\right\},
$$

where the max is taken over all possible choices of

$$
g_{i} \in\left\{z_{i} x-x_{i} z, z_{i} y-y_{i} z, x_{i} y-y_{i} x\right\} \subset \mathcal{O}_{L}[x, y, z], \quad i=1, \ldots, r .
$$

Now fix a choice of $g_{i} \in\left\{z_{i} x-x_{i} z, z_{i} y-y_{i} z, x_{i} y-y_{i} x\right\}, i=1, \ldots, r$. Since $\prod_{i=1}^{r} g_{i}$ vanishes at all the points $Q_{i}$, by the effective Hilbert Nullstellensatz (see Remark 26), there exists a positive integer $M$, homogeneous polynomials $a_{1}, a_{2} \in \mathcal{O}_{L}[x, y, z]$ with $\operatorname{deg} a_{1}=r M-\operatorname{deg} f_{1}, \operatorname{deg} a_{2}=r M-\operatorname{deg} f_{2}$, and a constant $a \in O_{L}$ such that

$$
f_{1}(x, y, z) a_{1}(x, y, z)+f_{2}(x, y, z) a_{2}(x, y, z)=a\left(\prod_{i=1}^{r} g_{i}\right)^{M}
$$

and

$$
\begin{aligned}
M & \leq(8 d)^{8}, \\
\log \max _{\substack{w \in M_{L} \\
w \mid \infty}}\left\{\left|a_{1}\right|_{w},\left|a_{2}\right|_{w},|a|_{w}\right\} & \leq(8 d)^{15}\left(h_{\infty}+8 d \log 8 d\right) .
\end{aligned}
$$

Let $w$ be a place of $L$ lying above $v$ (we will choose a specific such $w$ later). Let $x, y, z \in k$. It follows that there exists $a_{1}, a_{2}, a$, and $M$, as above, such that

$$
\begin{aligned}
\left(\prod_{i=1}^{r} \max \right. & \left\{\left|z_{i} x-x_{i} z\right|_{w},\left|z_{i} y-y_{i} z\right|_{w},\left|x_{i} y-y_{i} x\right|_{w}\right)^{M} \\
& =\frac{1}{|a|_{w}}\left|f_{1}(x, y, z) a_{1}(x, y, z)+f_{2}(x, y, z) a_{2}(x, y, z)\right|_{w} \\
& \leq 2 \max \left\{\left|f_{1}(x, y, z) a_{1}(x, y, z)\right|_{w},\left|f_{2}(x, y, z) a_{2}(x, y, z)\right|_{w}\right\} /|a|_{w} \\
& \leq 2(r M)^{2} \max _{i=1,2}\left\{\left|f_{i}(x, y, z)\right|_{w}\left|a_{i}\right|_{w} \max \left\{|x|_{w},|y|_{w},|z|_{w}\right\}^{r M-\operatorname{deg} f_{i}}\right\} /|a|_{w} .
\end{aligned}
$$

So

$$
\begin{aligned}
& \left(\prod_{i=1}^{r} \frac{\max \left\{\left|z_{i} x-x_{i} z\right|_{w},\left|z_{i} y-y_{i} z\right|_{w},\left|x_{i} y-y_{i} x\right|_{w}\right.}{\max \left\{|x|_{w},|y|_{w},|z|_{w}\right\}}\right)^{M} \\
& \quad \leq \frac{2(r M)^{2}}{|a|_{w}} \max \left\{\left|a_{1}\right|_{w},\left|a_{2}\right|_{w}\right\} \max _{i=1,2} \frac{\left|f_{i}(x, y, z)\right|_{w}}{\max \left\{|x|_{w},|y|_{w},|z|_{w}\right\}^{\operatorname{deg} f_{i}}} .
\end{aligned}
$$


Let $r_{w / v}=[L: k] /\left[L_{w}: k_{v}\right]$. Taking logarithms, rearranging, and using the definitions and inequalities above, we find

$$
\begin{array}{r}
\epsilon h(P) \leq \min \left\{h_{C_{1}, v}(P), h_{C_{2}, v}(P)\right\}=r_{w / v} \min \left\{h_{C_{1}, w}(P), h_{C_{2}, w}(P)\right\} \\
\leq M r_{w / v} \sum_{i=1}^{r} h_{Q_{i}, w}(P)-M r_{w / v} \sum_{i=1}^{r} \log \max \left\{\left\|x_{i}\right\|_{w},\left\|y_{i}\right\|_{w},\left\|z_{i}\right\|_{w}\right\} \\
\quad+\log 2(r M)^{2}+r_{w / v} \log \max \left\{\left\|f_{1}\right\|_{w},\left\|f_{2}\right\|_{w}\right\} \\
\quad+r_{w / v} \log \max \left\{\left\|a_{1}\right\|_{w},\left\|a_{2}\right\|_{w}\right\}-r_{w / v} \log \|a\|_{w} .
\end{array}
$$

Let $Q_{l} \in\left(C_{1} \cap C_{2}\right)(\bar{k})$. Then by assumption, there exists $i, j \in\{1, \ldots, n\}, i \neq j$, such that $Q_{l} \notin C_{i} \cup C_{j}$. Let $w_{l}$ be the place of $k\left(Q_{l}\right)$ lying below $w$ and let $r_{w_{l} / v}=\left[k\left(Q_{l}\right): k\right] /\left[k\left(Q_{l}\right)_{w_{l}}: k_{v}\right]$. Let $\Phi_{P}$ and $Z^{\prime}$ be as in Theorem 7.

By Theorem 37, either

$$
P \in \bigcap_{\phi \in \Phi_{Q_{l}}}\left\{Q \in X(\bar{k}): \phi(Q)=\phi\left(Q_{l}\right)\right\} \subset Z^{\prime}
$$

or

$$
\begin{aligned}
r_{w / v} h_{Q_{l}, w}(P) & =r_{w_{l} / v} h_{Q_{l}, w_{l}}(P) \\
& <\frac{\epsilon}{2 r M} h(P)+\max _{i, j} c_{4}\left(\frac{\epsilon}{2 r M r_{w_{l} / v}}, k, S, w_{l}, Q_{l}, C_{i}, C_{j}\right)
\end{aligned}
$$

for all $P \in\left(\mathbb{P}^{2} \backslash \bigcup_{i=1}^{n} C_{i}\right)\left(\mathbb{O}_{k, S}\right)=\bigcap_{i, j}\left(\mathbb{P}^{2} \backslash\left(C_{i} \cup C_{j}\right)\right)\left(\mathbb{O}_{k, S}\right)$.

Suppose now that $P \notin Z^{\prime}$. Summing over all points in $C_{1} \cap C_{2}$, we obtain

$$
M r_{w / v} \sum_{l=1}^{r} h_{Q_{l}, w}(P)<\frac{\epsilon}{2} h(P)+\sum_{l=1}^{r} \max _{i, j} c_{4}\left(\frac{\epsilon}{2 r M r_{w_{l} / v}}, k, S, w_{l}, Q_{l}, C_{i}, C_{j}\right) .
$$

Substituting into (8) we find that

$$
\begin{aligned}
h(P)<\frac{2}{\epsilon}\left(\sum_{l=1}^{r} \max _{i, j} c_{4}\left(\frac{\epsilon}{2 r M r_{w_{l} / v}}, k, S, w_{l}, Q_{l}, C_{i}, C_{j}\right)\right. \\
\quad+\log 2(r M)^{2}+r_{w / v} \log \max _{i=1,2}\left\|f_{i}\right\|_{w}+r_{w / v} \log \max _{i=1,2}\left\|a_{i}\right\|_{w} \\
\left.\quad-M r_{w / v} \sum_{i=1}^{r} \log \max \left\{\left\|x_{i}\right\|_{w},\left\|y_{i}\right\|_{w},\left\|z_{i}\right\|_{w}\right\}-r_{w / v} \log \|a\|_{w}\right) .
\end{aligned}
$$

We now estimate all the terms on the right-hand side. The dominant term, which comes from the first sum above, is

$$
\sum_{l=1}^{r} \max _{i, j} c_{5}\left(\frac{\epsilon}{2 r M r_{w_{l} / v}}, k, S, w_{l}, Q_{l}, C_{i}, C_{j}\right) .
$$


We estimate this term first. We note that by (2), (3), and (4),

$$
c_{6}(k, S) \leq 2^{3 s+3} s^{4 s-3} \delta^{2 s+2 \delta-5}\left(\log ^{*} N\right)^{2 s-\delta}|\Delta|\left(\log ^{*}|\Delta|\right)^{2 \delta-2} .
$$

Then

$$
\begin{aligned}
2 r M r_{w_{l} / v} d^{2} c_{2}\left(d^{2} \delta, s\right) N\left(w_{l}\right) c_{6}(k, S) & \\
& =72 r M d^{2} r_{w_{l} / v} N\left(w_{l}\right)\left(16 e d^{2} \delta\right)^{3 s+5}\left(\log ^{*} d^{2} \delta\right)^{2} c_{6}(k, S) \\
& \leq 2^{20 s+61} d^{6 s+28} \delta^{5 s+2 \delta+2} s^{4 s-3} N^{d^{2}}\left(\log ^{*} N\right)^{2 s-\delta}|\Delta|\left(\log ^{*}|\Delta|\right)^{2 \delta-2} .
\end{aligned}
$$

Simple estimates then also give

$$
\log 2 r M r_{w_{l} / v} d^{2} c_{2}\left(d^{2} \delta, s\right) N\left(w_{l}\right) c_{6}(k, S) / \epsilon \leq 2^{7} s^{2} d^{2}\left(\log ^{*} N\right)\left(\log ^{*}|\Delta|\right) / \epsilon .
$$

We have $\sum_{l=1}^{r} h\left(Q_{l}\right) \leq d\left(h\left(C_{1}\right)+h\left(C_{2}\right)+4 d\right) \leq 4 d^{2}(h+1)$, by Theorem 27 , and

$$
\begin{aligned}
\sum_{l=1}^{r} \max _{i, j} & c_{7}\left(k, Q_{l}, C_{i}, C_{j}\right) \\
& \leq \sum_{l=1}^{r}\left(d^{2} h\left(Q_{l}\right)+2 d h+2 d^{2} \log 2+2 \log \left(d^{2}+2\right)+\frac{1}{\delta} \log |\Delta|+2 \delta c_{3}(k)\right) \\
& \leq 2^{4 \delta+2} \delta^{6 \delta-5} \sqrt{|\Delta|}\left(\log ^{*}|\Delta|\right)^{\delta} d^{4}(h+1) .
\end{aligned}
$$

Then (10) is bounded by

$$
2^{20 s+4 \delta+73} d^{6 s+34} \delta^{5 s+8 \delta-3} s^{4 s-1} N^{d^{2}}\left(\log ^{*} N\right)^{2 s}|\Delta|^{3 / 2}\left(\log ^{*}|\Delta|\right)^{3 \delta}(h+1) / \epsilon^{2} .
$$

In the remainder of the proof, we will show that the sum of the remaining elements in the parentheses on the right-hand side of (9) can also be bounded by this quantity. Thus, we find that $h(P) \leq 2^{20 s+4 \delta+75} d^{6 s+34} \delta^{5 s+8 \delta-3} s^{4 s-1} N^{d^{2}}\left(\log ^{*} N\right)^{2 s}|\Delta|^{3 / 2}\left(\log ^{*}|\Delta|\right)^{3 \delta}(h+1) / \epsilon^{3}$, proving the lemma.

First, we handle the remaining terms coming from the first sum in (9):

$$
\begin{aligned}
& \begin{aligned}
& \sum_{l=1}^{r}\left(2 d^{2}+2\right) h\left(Q_{l}\right)+10 r \log \left(d^{2}+\right.2)+\left(2 d^{2}+7\right) r \log 2 \\
&+ \frac{r}{d}\left(h\left(C_{1}\right)+h\left(C_{2}\right)\right)+\frac{r}{\delta d^{2}} \log |\Delta|+\frac{2 \delta r}{d^{2}} c_{3}(k) \\
& \leq 4\left(2 d^{2}+2\right) d^{2}(h+1)+10 d^{2} \log \left(d^{2}+2\right)+\left(2 d^{2}+7\right) d^{2} \\
&+ 2 d h+\frac{1}{\delta} \log |\Delta|+2 \delta 2^{4 \delta} \delta^{6 \delta} \sqrt{|\Delta|}\left(\log ^{*}|\Delta|\right)^{\delta} \\
& \leq 55 d^{4}(h+1)+2^{4 \delta+2} \delta^{6 \delta+1} \sqrt{|\Delta|}\left(\log ^{*}|\Delta|\right)^{\delta} .
\end{aligned}
\end{aligned}
$$


We now bound $h_{\infty}$, after making some appropriate choices. Choose $f_{1}, f_{2}$, and $Q_{l}=\left(x_{l}, y_{l}, z_{l}\right), l=1, \ldots, r$, as in Lemma 30(b). Then

$$
\begin{aligned}
-\left(\left(d^{2} \delta\right)^{2} h\left(Q_{l}\right)+\frac{\left(d^{2} \delta\right)}{2} \log \left(d^{2} \delta\right)\right) & \leq \log \max \left\{\left\|x_{l}\right\|_{w_{l}},\left\|y_{l}\right\|_{w_{l}},\left\|z_{l}\right\|_{w_{l}}\right\} \\
& \leq\left(2 d^{2} \delta+1\right) h\left(Q_{l}\right)+\log d^{2} \delta, \\
\log \max _{\substack{v \in M_{k} \\
v \mid \infty}}\left|f_{1}\right|_{v} & \leq(2 \delta+1) \delta h\left(C_{1}\right)+\delta \log \delta \leq 3 \delta^{2}(h+1), \\
\log \max _{\substack{v \in M_{k} \\
v \mid \infty}}\left|f_{2}\right|_{v} & \leq(2 \delta+1) \delta h\left(C_{2}\right)+\delta \log \delta \leq 3 \delta^{2}(h+1) .
\end{aligned}
$$

Let $g_{l} \in\left\{z_{l} x-x_{l} z, z_{l} y-y_{l} z, x_{l} y-y_{l} x\right\}, i=1, \ldots, r$. If $w \in M_{L}, w \mid \infty$, then

$$
\begin{aligned}
\log \left|\prod_{l=1}^{r} g_{l}\right|_{w} & \leq \log 2^{r} \prod_{l=1}^{r}\left|g_{l}\right|_{w} \leq r \log 2+\log \sum_{l=1}^{r}\left|g_{l}\right|_{w} \\
& \leq r \log 2+\sum_{l=1}^{r} \log \max \left\{\left|x_{l}\right|_{w_{l}},\left|y_{l}\right|_{w_{l}},\left|z_{l}\right|_{w_{l}}\right\} \\
& \leq d^{2} \log 2+\sum_{l=1}^{r}\left(\left(2 d^{2} \delta+1\right) d^{2} \delta h\left(Q_{l}\right)+d^{2} \delta \log d^{2} \delta\right) \\
& \leq d^{2} \log 2+4\left(2 d^{2} \delta+1\right) d^{4} \delta(h+1)+d^{4} \delta \log d^{2} \delta .
\end{aligned}
$$

Since $w \in M_{L}$ was arbitrary, we have

$$
\log \max _{\substack{w \in M_{L} \\ w \mid \infty}}\left|\prod_{l=1}^{r} g_{l}\right|_{w} \leq d^{2} \log 2+4\left(2 d^{2} \delta+1\right) d^{4} \delta(h+1)+d^{4} \delta \log d^{2} \delta
$$

It follows easily that

$$
h_{\infty} \leq 14 d^{6} \delta^{2}(h+1) .
$$

Then from the above, we have

$$
\log 2(r M)^{2} \leq \log 2 d^{4}(8 d)^{16} \leq 2^{6} d,
$$

$r_{w / v} \log \max \left\{\left\|f_{1}\right\|_{w},\left\|f_{2}\right\|_{w}\right\} \leq \log \max \left\{\left|f_{1}\right|_{w},\left|f_{2}\right|_{w}\right\} \leq 4 \delta^{2}(h+1)$,

$r_{w / v} \log \max \left\{\left\|a_{1}\right\|_{w},\left\|a_{2}\right\|_{w}\right\} \leq \log \max \left\{\left|a_{1}\right|_{w},\left|a_{2}\right|_{w}\right\} \leq(8 d)^{15}\left(h_{\infty}+8 d \log 8 d\right)$

$$
\leq 2^{51} d^{21} \delta^{2}(h+1) \text {. }
$$


We also find

$$
\begin{aligned}
-M r_{w / v} \sum_{l=1}^{r} \log \max \left\{\left\|x_{l}\right\|_{w},\left\|y_{l}\right\|_{w},\left\|z_{l}\right\|_{w}\right\} & \\
& =-M \sum_{l=1}^{r} r_{w_{l} / v} \log \max \left\{\left\|x_{l}\right\|_{w_{l}},\left\|y_{l}\right\|_{w_{l}},\left\|z_{l}\right\|_{w_{l}}\right\} \\
& \leq(8 d)^{8} d^{2}\left(\left(d^{2} \delta\right)^{2} \sum_{l=1}^{r} h\left(Q_{l}\right)+\frac{d^{4} \delta}{2} \log \left(d^{2} \delta\right)\right) \\
& \leq 2^{24} d^{10}\left(4 d^{6} \delta^{2}(h+1)+\frac{d^{4} \delta}{2} \log \left(d^{2} \delta\right)\right) \\
& \leq 2^{27} d^{16} \delta^{2}(h+1) .
\end{aligned}
$$

From the product formula and the fact that $a \in O_{L}$, we have the inequality

$$
\begin{aligned}
-\sum_{\substack{w^{\prime} \in M_{L} \\
w^{\prime} \mid v}} \log \|a\|_{w^{\prime}} & =\sum_{\substack{w^{\prime} \in M_{L} \\
w^{\prime} \nmid v}} \log \|a\|_{w^{\prime}} \leq \sum_{\substack{w^{\prime} \in M_{L} \\
w^{\prime} \mid \infty}} \max \left\{\log \|a\|_{w^{\prime}}, 0\right\} \\
& \leq(8 d)^{15}\left(h_{\infty}+8 d \log 8 d\right) \leq 2^{51} d^{21} \delta^{2}(h+1) .
\end{aligned}
$$

Since $L / k$ is Galois, there are exactly $r_{w / v}$ places $w^{\prime} \in M_{L}$ with $w^{\prime} \mid v$. Therefore, there exists a place $w^{\prime} \in M_{L}$ with $w^{\prime} \mid v$ and $-\log \|a\|_{w^{\prime}} \leq\left(1 / r_{w / v}\right) 2^{51} d^{36} \delta^{2}(h+1)$. Choosing now $w=w^{\prime}$, we have

$$
-r_{w / v} \log \|a\|_{w} \leq 2^{51} d^{21} \delta^{2}(h+1) .
$$

Summing all of the inequalities above, we find that, as claimed, the remaining terms in (9) are easily bounded by (11).

\section{Acknowledgments}

The author would like to thank Mike Bennett for sharing and discussing his results.

\section{References}

[Autissier 2009] P. Autissier, "Géométries, points entiers et courbes entières", Ann. Sci. Éc. Norm. Supér. (4) 42:2 (2009), 221-239. MR 2010j:14044 Zbl 1173.14016

[Autissier 2011] P. Autissier, "Sur la non-densité des points entiers", Duke Math. J. 158:1 (2011), 13-27. MR 2012j:14033 Zbl 1217.14020

[Baker 1975] A. Baker, Transcendental number theory, Cambridge University Press, 1975. MR 54 \#10163 Zbl 0297.10013

[Bérczes et al. 2009] A. Bérczes, J.-H. Evertse, and K. Győry, "Effective results for linear equations in two unknowns from a multiplicative division group", Acta Arith. 136:4 (2009), 331-349. MR 2009j:11054 Zbl 1234.11034

[Bilu 1995] Y. Bilu, "Effective analysis of integral points on algebraic curves", Israel J. Math. 90:1-3 (1995), 235-252. MR 96e:11082 Zbl 0840.11028 
[Bombieri 1983] E. Bombieri, "On Weil's "théorème de décomposition"”, Amer. J. Math. 105:2 (1983), 295-308. MR 84g:14029 Zbl 0516.12009

[Bugeaud and Győry 1996] Y. Bugeaud and K. Győry, "Bounds for the solutions of unit equations", Acta Arith. 74:1 (1996), 67-80. MR 97b:11045 Zbl 0861.11023

[Corvaja and Zannier 2004] P. Corvaja and U. Zannier, “On integral points on surfaces”, Ann. of Math. (2) 160:2 (2004), 705-726. MR 2005m:11118 Zbl 1146.11035

[Corvaja and Zannier 2006] P. Corvaja and U. Zannier, "On the integral points on certain surfaces", Int. Math. Res. Not. 2006 (2006), Art. ID 98623. MR 2006m:14030 Zbl 1145.14023

[Corvaja et al. 2009] P. Corvaja, A. Levin, and U. Zannier, "Integral points on threefolds and other varieties”, Tohoku Math. J. (2) 61:4 (2009), 589-601. MR 2011a:11125 Zbl 1250.11066

[Evertse 1984] J.-H. Evertse, "On sums of $S$-units and linear recurrences”, Compositio Math. 53:2 (1984), 225-244. MR 86c:11045 Zbl 0547.10008

[Hajdu 1993] L. Hajdu, “A quantitative version of Dirichlet's $S$-unit theorem in algebraic number fields”, Publ. Math. Debrecen 42:3-4 (1993), 239-246. MR 94e:11118 Zbl 0798.11051

[Hindry and Silverman 2000] M. Hindry and J. H. Silverman, Diophantine geometry: An introduction, Graduate Texts in Mathematics 201, Springer, New York, 2000. MR 2001e:11058 Zbl 0948.11023

[Lenstra 1992] H. W. Lenstra, Jr., "Algorithms in algebraic number theory", Bull. Amer. Math. Soc. (N.S.) 26:2 (1992), 211-244. MR 93g:11131 Zbl 0759.11046

[Levin 2008] A. Levin, "Variations on a theme of Runge: Effective determination of integral points on certain varieties", J. Théor. Nombres Bordeaux 20:2 (2008), 385-417. MR 2009j:11104 Zbl 1179.11018

[Levin 2009] A. Levin, “Generalizations of Siegel's and Picard's theorems”, Ann. of Math. (2) 170:2 (2009), 609-655. MR 2010k:11116 Zbl 1250.11067

[Masser 1988] D. W. Masser, "Linear relations on algebraic groups", pp. 248-262 in New advances in transcendence theory (Durham, 1986), edited by A. Baker, Cambridge Univ. Press, 1988. MR 89j:11065 Zbl 0656.10031

[Masser and Wüstholz 1983] D. W. Masser and G. Wüstholz, "Fields of large transcendence degree generated by values of elliptic functions", Invent. Math. 72:3 (1983), 407-464. MR 85g:11060 Zbl 0516.10027

[Mo and Tijdeman 1992] D. Z. Mo and R. Tijdeman, "Exponential Diophantine equations with four terms", Indag. Math. (N.S.) 3:1 (1992), 47-57. MR 93d:11035 Zbl 0765.11018

[Philippon 1995] P. Philippon, "Sur des hauteurs alternatives, III", J. Math. Pures Appl. (9) 74:4 (1995), 345-365. MR 97a:11098 Zbl 0878.11025

[van der Poorten and Schlickewei 1982] A. J. van der Poorten and H. P. Schlickewei, "The growth conditions for recurrence sequences”, Macquarie Univ. Math. Rep. 82-0041, Macquarie University, 1982.

[Runge 1887] C. Runge, "Über ganzzahlige Lösungen von Gleichungen zwischen zwei Veränderlichen”, J. Reine Angew. Math. 100 (1887), 425-435. JFM 19.0076.03

[Schmidt 1991] W. M. Schmidt, "Construction and estimation of bases in function fields", J. Number Theory 39:2 (1991), 181-224. MR 93b:11079 Zbl 0764.11046

[Silverman 1984] J. H. Silverman, "Lower bounds for height functions", Duke Math. J. 51:2 (1984), 395-403. MR 87d:11039 Zbl 0579.14035

[Silverman 1987] J. H. Silverman, "Arithmetic distance functions and height functions in Diophantine geometry”, Math. Ann. 279:2 (1987), 193-216. MR 89a:11066 Zbl 0607.14013 
[Skinner 1990] C. M. Skinner, "On the Diophantine equation $a p^{x}+b q^{v}=c+d p^{z} q^{w}$ ", J. Number Theory 35:2 (1990), 194-207. MR 91h:11021 Zbl 0703.11017

[Vojta 1983] P. A. Vojta, Integral points on varieties, Ph.D. thesis, Harvard University, 1983, Available at http://search.proquest.com/docview/303162150.

[Vojta 1987] P. Vojta, Diophantine approximations and value distribution theory, Lecture Notes in Mathematics 1239, Springer, Berlin, 1987. MR 91k:11049 Zbl 0609.14011

Communicated by Joseph Silverman

Received 2013-04-14 Revised 2013-09-02 Accepted 2013-10-02

adlevin@math.msu.edu Department of Mathematics, Michigan State University, 619 Red Cedar Road, East Lansing, MI 48824, United States 


\section{Algebra \& Number Theory}

msp.org/ant

\section{EDITORS}

MANAGING EDITOR

Bjorn Poonen

Massachusetts Institute of Technology

Cambridge, USA

\author{
EDITORIAL BOARD CHAIR \\ David Eisenbud \\ University of California \\ Berkeley, USA
}

\section{BOARD OF EDITORS}

Georgia Benkart

Dave Benson

Richard E. Borcherds

John H. Coates

J-L. Colliot-Thélène

Brian D. Conrad

Hélène Esnault

Hubert Flenner

Edward Frenkel

Andrew Granville

Joseph Gubeladze

Roger Heath-Brown

Ehud Hrushovski

Craig Huneke

Mikhail Kapranov

Yujiro Kawamata

János Kollár

Yuri Manin

Barry Mazur

Philippe Michel
University of Wisconsin, Madison, USA

University of Aberdeen, Scotland

University of California, Berkeley, USA

University of Cambridge, UK

CNRS, Université Paris-Sud, France

University of Michigan, USA

Freie Universität Berlin, Germany

Ruhr-Universität, Germany

University of California, Berkeley, USA

Université de Montréal, Canada

San Francisco State University, USA

Oxford University, UK

Hebrew University, Israel

University of Virginia, USA

Yale University, USA

University of Tokyo, Japan

Princeton University, USA

Northwestern University, USA

Harvard University, USA

École Polytechnique Fédérale de Lausanne
Susan Montgomery

Shigefumi Mori

Raman Parimala

Jonathan Pila

Victor Reiner

Karl Rubin

Peter Sarnak

Joseph H. Silverman

Michael Singer

Vasudevan Srinivas

J. Toby Stafford

Bernd Sturmfels

Richard Taylor

Ravi Vakil

Michel van den Bergh

Marie-France Vignéras

Kei-Ichi Watanabe

Efim Zelmanov

Shou-Wu Zhang
University of Southern California, USA

RIMS, Kyoto University, Japan

Emory University, USA

University of Oxford, UK

University of Minnesota, USA

University of California, Irvine, USA

Princeton University, USA

Brown University, USA

North Carolina State University, USA

Tata Inst. of Fund. Research, India

University of Michigan, USA

University of California, Berkeley, USA

Harvard University, USA

Stanford University, USA

Hasselt University, Belgium

Université Paris VII, France

Nihon University, Japan

University of California, San Diego, USA

Princeton University, USA

PRODUCTION

production@msp.org

Silvio Levy, Scientific Editor

See inside back cover or msp.org/ant for submission instructions.

The subscription price for 2014 is US $\$ 225 /$ year for the electronic version, and $\$ 400 /$ year $(+\$ 55$, if shipping outside the US) for print and electronic. Subscriptions, requests for back issues and changes of subscribers address should be sent to MSP.

Algebra \& Number Theory (ISSN 1944-7833 electronic, 1937-0652 printed) at Mathematical Sciences Publishers, 798 Evans Hall \#3840, c/o University of California, Berkeley, CA 94720-3840 is published continuously online. Periodical rate postage paid at Berkeley, CA 94704, and additional mailing offices.

ANT peer review and production are managed by EditFLOW ${ }^{\circledR}$ from Mathematical Sciences Publishers.

\section{PUBLISHED BY}

- mathematical sciences publishers

nonprofit scientific publishing

http://msp.org/

(C) 2014 Mathematical Sciences Publishers 


\section{Algebra \& Number Theory}

Volume $8 \quad$ No. $3 \quad 2014$

Derived invariants of irregular varieties and Hochschild homology

LUIGI LOMBARDI

Sato-Tate distributions of twists of $y^{2}=x^{5}-x$ and $y^{2}=x^{6}+1$

FRANCESC Fité and ANDREW V. SUTHERLAND

The algebraic dynamics of generic endomorphisms of $\mathbb{P}^{n}$

NAJMUDDIN FAKHRUDDIN

The tame-wild principle for discriminant relations for number fields

JOHN W. JONES and DAVID P. ROBERTS

Linear forms in logarithms and integral points on higher-dimensional varieties

AARON LEVIN

Lefschetz theorem for abelian fundamental group with modulus

689

MORITZ KERZ and ShuJi SAITO

Localization of spherical varieties

703

FRIEDRICH KNOP

Lefschetz operator and local Langlands modulo $\ell$ : the limit case

JEAN-FRANÇOIS DAT

Splitting tower and degree of tt-rings

PAUL BALMER 